\title{
Organisationaler Wandel als Bedrohung - von impliziter Angst zur Annäherung durch prozedurale Gerechtigkeit
}

\author{
Stefan Reiss ${ }^{1}$ D $\cdot$ Liza Prentice ${ }^{1} \cdot$ Christoph Schulte-Cloos $^{1}$ Eva Jonas ${ }^{1}$ \\ Online publiziert: 22. Mai 2019 \\ (c) Der/die Autor(en) 2019
}

\section{Zusammenfassung}

In der gegenwärtigen Arbeitswelt müssen Organisationen in der Lage sein, sich schnell auf neue Marktanforderungen anzupassen. Mitarbeiter werden jedoch von organisationalem Wandel oft belastet. In diesem Artikel beschäftigen wir uns mit den impliziten negativen emotionalen und motivationalen Reaktionen auf organisationalen Wandel sowie möglichen Wegen, diese Schwierigkeiten zu bewältigen. In Studie $1(n=76)$ führte das Empfinden von Bedürfnis- und Erwartungsdiskrepanzen im Rahmen von organisationalem Wandel zu selbstberichtetem körperlichem Arousal, das mit Ängstlichkeit und vermindertem Annäherungs-Affekt korrelierte. Je stärker die Maßnahme als Diskrepanz wahrgenommen wurde, desto negativer waren die emotionalen Auswirkungen. Eine Intervention zur Erhöhung prozeduraler Gerechtigkeit reduzierte diese negativen Effekte und führte zu signifikanter Abnahme von körperlicher Aktivierung und zu stärkerer Aktivierung von Annäherungs-Affekt. In Studie $2(n=125)$ erforschten wir eingehender die Einflüsse von prozeduraler Gerechtigkeit und dem Framing einer Veränderungsmaßnahme auf Reaktionen auf organisationalen Wandel. Probanden, die ein annäherungsformuliertes Szenario lasen, das die Möglichkeit eigener Meinungsäußerung beinhaltete, zeigten verringerte implizite Vermeidungsmotivation und weniger negativen ängstlichen Affekt im Vergleich zu Probanden, die ein vermeidungsformuliertes Szenario lasen und keine Möglichkeit zur Meinungsäußerung hatten. Der Effekt war besonders ausgeprägt bei MitarbeiterInnen mit regulatorischem Promotion-Fokus. Die Ergebnisse deuten darauf hin, dass organisationaler Wandel als Bedrohung wahrgenommen werden kann. Durch prozedurale Gerechtigkeit und die Formulierung der Maßnahme in Annäherungskontext können MitarbeiterInnen jedoch dabei unterstützt werden, wieder in die Handlungsfähigkeit zu kommen.

Schlüsselwörter Vermeidungsmotivation · Annäherungsmotivation · Psychologische Bedrohung · Regulatorischer Fokus . Prozedurale Gerechtigkeit

Zusatzmaterial online Zusätzliche Informationen sind in der Online-Version dieses Artikels (https://doi.org/10.1007/s11612019-00469-x) enthalten.

Stefan Reiss, MSc.

stefan.reiss@sbg.ac.at

1 Fachbereich Psychologie, Universität Salzburg,

Hellbrunnerstr. 34, 5020 Salzburg, Österreich 


\title{
Organizational change as threat - from implicit anxiety to approach through procedural justice
}

\begin{abstract}
In today's working environment, organizations need to be able to adapt quickly to new market requirements. However, employees often feel burdened by organizational change. In this article, we examine the implicit negative emotional and motivational responses to organizational change and possible ways to overcome these difficulties. In Study $1(n=76)$, the perception of need- and expectation discrepancies led to self-reported physiological arousal which correlated with anxiety and reduced approach affect. The more the change measure was perceived as a discrepancy, the more negative were the emotional outcomes. An intervention to increase procedural justice reduced these negative effects: it led to a decrease in physiological activation and an increase in approach-affect. In Study $2(n=125)$, we investigated in more detail the influences of procedural justice and framing on reactions to organizational change. Participants who read an approach-framed scenario including the possibility to express their own opinions showed reduced implicit avoidance motivation and less negative anxious affect compared to participants who read an avoidance-framed scenario without being able to express their opinions. This effect was particularly pronounced among employees with regulatory promotion focus. The results indicate that organizational change can be perceived as a threat. However, procedural justice and approach-framing of organizational change can help employees to regain approach motivation.
\end{abstract}

Keywords Avoidance motivation - Approach motivation $\cdot$ Psychological threat $\cdot$ Regulatory focus $\cdot$ Procedural justice

\section{Organisationaler Wandel als Bedrohung}

In der gegenwärtigen Arbeitswelt können Unternehmen ihr Überleben nur durch kontinuierlichen Wandel sichern: Externe Einflüsse wie wirtschaftliche Bedingungen, neue Technologien, Digitalisierung, neue Vorlieben bestimmter Kundengruppen, politische oder gesetzliche Maßnahmen oder gesellschaftlicher Wertewandel zwingen Unternehmen zu permanenten Anpassungen und strategischem Umdenken (Lewis 2019; Palmer et al. 2017). Erfolgreicher Wandel verschafft dem Unternehmen Wettbewerbsvorteile, besseres Kostenmanagement, und dezentralisierte Kontrolle (Leana und Barry 2000).

Doch obwohl die Wichtigkeit von Veränderungsmaßnahmen allseits bekannt ist, kann Wandel riskant sein (Jacobs et al. 2013). Immer wieder scheitert Wandel im Durchführungsprozess, wie die Zahlen für gescheiterte Veränderungsmaßnahmen, die je nach Quelle zwischen $60 \%$ und bis zu $90 \%$ variieren, veranschaulichen (Balogun und Hailey 2008; Burnes 2009; für eine aktuelle Übersicht siehe Lewis 2019; Stouten et al. 2018). Auch wenn die Ursachenfindung oft schwierig ist, so ist unumstritten, dass der Erfolg von Veränderungsmaßnahmen zentral von den involvierten MitarbeiterInnen und deren Bereitschaft für den Wandel abhängt (Scholz 2015). In den vergangenen Jahren hat sich die Forschung mit den psychologischen Auswirkungen von organisationalem Wandel auf Mitarbeiter beschäftigt, und dabei die Wichtigkeit von Emotionen erkannt (z.B. Vuori und Virtaharju 2012). Studien zu diesem Thema zeigten, dass Veränderungsprozesse oft zu negativen Emotionen wie Ängstlichkeit und Verunsicherung oder zu emotionalem Rückzug führen (z. B. Eriksson 2004; Kiefer 2002; Schumacher et al. 2016). Diese können sich wiederum negativ auf die Unterstützung der Maßnahme auswirken und Widerstand hervorrufen (Helpap und Bekmeier-Feuerhahn 2016). Somit können diese emotionalen Erfahrungen Auswirkungen auf die Motivation von MitarbeiterInnen haben, einen Veränderungsprozess aktiv zu unterstützen, was wiederum Einfluss auf den Erfolg der Maßnahme und des Unternehmens nehmen kann.

In diesem Artikel wollen wir genau diese emotionalen und motivationalen Prozesse untersuchen. Wir nehmen an, dass organisationaler Wandel als psychologische Bedrohung wahrgenommen werden und so Angst und negative Emotionen auslösen kann. Zusätzlich zu diesen emotionalen Auswirkungen kann Bedrohung auf motivationaler Ebene auch zu einer Hemmung des Verhaltens von MitarbeiterInnen führen, was den Erfolg von Maßnahmen in der Umsetzung gefährden kann. Wir betten diese Betrachtungsweise in den theoretischen Kontext der sozialpsychologischen Bedrohungsforschung (Jonas et al. 2014) ein. Wir glauben, dass diese Betrachtungsweise unser Verständnis für den Erfolg von Veränderungsprozessen fördern kann. Außerdem wollen wir untersuchen, welche Interventionen dazu beitragen können, mit dem Wandel verbundene Ängste zu reduzieren und die positive motivationale Orientierung von MitarbeiterInnen zu fördern.

\subsection{Ein Fallbeispiel}

Nehmen wir als Fallbeispiel Frau A, eine langjährige Mitarbeiterin im Vertrieb eines IT-Unternehmens. Durch eine Umstrukturierung in ihrer Abteilung sollen betriebliche Abläufe effizienter gestaltet werden, was für Frau A mit einem veränderten Tätigkeitsfeld, neuen Aufgaben und neuen Funktionsbereichen einhergeht. Frau A hat ihr gewohntes 
Abb. 1 Schematische Darstellung des Prozesses von Bedrohung zu Annäherung im Kontext organisationalen Wandels

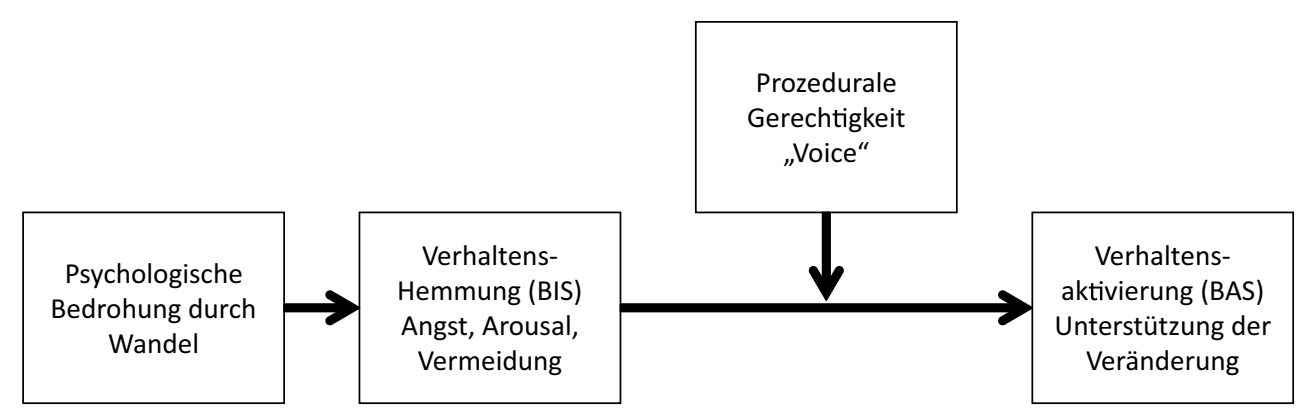

Arbeitsumfeld, ihr Team und ihre Tätigkeiten bisher sehr wertschätzt und ist nun verunsichert, weil sie befürchtet, mit den neuen Anforderungen nicht zurechtzukommen.

\subsection{Von der Hemmung zur Annäherung}

Wandel in Organisationen kann den Arbeitsalltag von Angestellten grundlegend und nachhaltig verändern: Gewohnte Arbeitsabläufe ändern sich, für manche MitarbeiterInnen stehen Arbeitsplatzsicherheit, soziale Beziehungen zu KollegInnen und Vorgesetzten oder Aufstiegsmöglichkeiten in Frage (vgl. Fugate et al. 2012). Viele MitarbeiterInnen empfinden geplante Veränderungen als Stressoren (Lee et al. 2017) und begegnen dieser Gefährdung des Status quo deshalb mit Unbehagen oder gar psychosomatischen Beschwerden (Schumacher et al. 2016). Solche Diskrepanzen zwischen Ist- und Soll-Zustand, also z. B. einer aktuell wahrgenommenen Situation von Unsicherheit und Risiko und einem gewünschten Zustand von Sicherheit und $\mathrm{Zu}$ versicht, definieren laut dem generellen Prozessmodell von Bedrohung und Verteidigung (Jonas et al. 2014) psychologische Bedrohung. Wenn Personen solchen Diskrepanzen begegnen, wägen sie die ihnen verfügbaren Ressourcen und die Ansprüche der Situation an ihre Ressourcen ab. Wenn eigene Ressourcen überwiegen und die Situation als kontrollierbar erlebt wird, reagieren Personen typischerweise mit einem Gefühl der Herausforderung, und der Organismus stellt Energie für das Verfolgen von Zielen zur Verfügung. Reichen jedoch die eigenen Ressourcen nicht aus, um die situativen Anforderungen zu bewältigen, erscheint die Situation als nicht kontrollierbar und wird als Bedrohung interpretiert (Blascovich 2008).

Bedrohung kann durch Diskrepanzen zwischen persönlichen Bedürfnissen oder Motiven und der Realität entstehen, zum Beispiel wenn Menschen gebeten werden, gewohnte Verhaltensweisen zu verändern, an denen sie aber gerne festhalten würden (Klonek et al. 2015). Eine andere Quelle für Diskrepanzen kann zwischen der Realität und den Erwartungen einer Person entstehen, zum Beispiel wenn unerwartet die eigene Handlungsfreiheit eingeschränkt wird (Sittenthaler et al. 2015; Steindl und Jonas 2015). Auch bereits kleinere Veränderungsmaßnahmen im Kontext von organisationalem Wandel können für Personen als Bedrohungen wahrgenommen werden und mit Angsterleben assoziierte neuronale Prozesse aktivieren. Frau A in unserem Beispiel würde die Situation also als Bedrohung wahrnehmen, weil sie den Status quo sehr wertgeschätzt hat und befürchtet, mit der veränderten Situation nicht gut zurecht zu kommen.

Laut dem generellen Prozessmodel von Bedrohung und Verteidigung (Jonas et al. 2014) wird bei psychologischer Bedrohung aktuelles, zielgerichtetes Verhalten unterbrochen und ein Zustand von Verhaltenshemmung (behavioraler Inhibition) ausgelöst, der durch das behaviorale Inhibitionssystem (BIS; Gray 1981) mediiert wird. Dieser Zustand manifestiert sich durch Vermeidungsmotivation, körperliche Erregung (Arousal), Ängstlichkeit und erhöhte Aufmerksamkeit (Vigilanz) (Corr 2004; Gray und McNaughton 2000). Ein neurophysiologisches Symptom von BIS-Aktivierung ist ein Anstieg rechtshemisphärischer Aktivität im präfrontalen Cortex (Agroskin et al. 2016; Amodio et al. 2008). Diese rechtsseitige Asymmetrie steht mit Verhaltenshemmung, passiver Vermeidung, und reduzierter Annäherungsmotivation in Verbindung (Agroskin et al. 2016; Angus und Harmon-Jones 2016). In Folge sinkt bei Frau A die Bereitschaft, den Wandel aktiv zu verfolgen.

Um diesen Zustand zu beenden und wieder Handlungsfähigkeit zu erlangen, muss Frau A das behaviorale Annäherungssystem (BAS; Gray 1981) aktivieren, das zielgerichtetes Verhalten ermöglicht. Im Gegensatz zum BIS ist BAS-Aktivierung durch einen Anstieg von linkshemisphärischer Aktivität im präfrontalen Cortex gekennzeichnet (E. Harmon-Jones et al. 2010). Diese linksseitige Asymmetrie steht in Verbindung mit Annäherungsmotivation und Verhaltensaktivierung, sowohl mit Freude als auch mit negativer Annäherung wie Wut und Ärger (Angus und Harmon-Jones 2016; E. Harmon-Jones und Allen 1998). Im Fall von Frau A kann diese Annäherung positiv sein, im Sinne von Unterstützung für die Maßnahme, aber auch destruktiv, zum Beispiel durch Protest oder Widerstand gegen die Veränderung. Aufgrund der Bedrohung von Frau As Bedürfnissen und der Verletzungen ihrer Erwartungen durch die Veränderungsmaßnahme, erwarten wir im Sinne des Bedrohungsmodells zwei Resultate: wenn sie die Veränderung 
als Bedrohung wahrnimmt, sollte Frau A zum einen stärkeren BIS-bezogenen Affekt wie Ängstlichkeit und reduzierten BAS-bezogenen Affekt wie Entschlossenheit oder Entspanntheit empfinden, und in einen Zustand der Verhaltenshemmung kommen. Zum anderen erwarten wir, dass sie zum Auflösen des entstandenen BIS-Zustands weniger unterstützend, sondern viel mehr gegen die Veränderungsmaßnahme eingestellt handelt.

Wir möchten uns organisationalem Wandel als möglicher Quelle von Bedrohung widmen, in dem wir untersuchen, ob die Konfrontation mit organisationalem Wandel zu BIS-Aktivierung führt. Um diese negativen Effekte zu lindern, untersuchen wir in einem zweiten Schritt Interventionen, die prozedurale Gerechtigkeit erhöhen und damit helfen, ihre Bedürfnisse zu berücksichtigen, BIS-Aktivierung zu reduzieren und BAS-Aktivierung zu erhöhen. Auf diese Weise soll Unterstützung für die Veränderung erhalten werden. Wir vermuten also, dass prozedurale Gerechtigkeit dazu beitragen kann, dass Personen ihre angstbezogene Hemmung überwinden indem sie den Wandel unterstützen, anstatt sich ihm entgegenzustellen (vgl. Cohen-Charash und Spector 2001; Abb. 1).

\subsection{Prozedurale Gerechtigkeit}

Vergangene Forschung konnte belegen, dass erfolgreicher Wandel in Organisationen auch davon abhängt, dass auf die Mitglieder der Organisation geachtet wird (Al-Haddad und Kotnour 2015; van den Heuvel et al. 2014). Eine zentrale Rolle spielt hier die Wahrnehmung von organisationaler Gerechtigkeit.

Die Literatur beschreibt vier Dimensionen organisationaler Gerechtigkeit (Colquitt 2001): Distributive Gerechtigkeit wird als gerechte Verteilung von Aufgaben und Belohnungen definiert, prozedurale Gerechtigkeit beschreibt die Möglichkeit von Personen, Prozesse oder Ergebnisse $\mathrm{zu}$ beeinflussen. Interpersonale Gerechtigkeit beinhaltet respektvolle und angemessene Behandlung; informationale Gerechtigkeit umfasst rechtzeitigen und zuverlässigen Informationsfluss. Zu Beginn der 2000er Jahre erschienen mehrere Meta-Analysen, welche die positiven Wirkungen von allen Gerechtigkeitsdimensionen in Organisationen zusammenfassten (Cohen-Charash und Spector 2001; Colquitt et al. 2001; Viswesvaran und Ones 2002). Organisationale Gerechtigkeit dient als Puffer gegen Stressoren, zum Beispiel wenn berufliche Anforderungen steigen (Proost et al. 2015). Im Kontext von Veränderungsprozessen wurde organisationale Gerechtigkeit als Intervention schon im letzten Jahrhundert vorgestellt (z.B. Folger et al. 1999). Empirische Studien bestätigen eine positive Verbindung zwischen den vier Gerechtigkeitsdimensionen und der Unterstützung von organisationalem Wandel (Fuchs 2011; Shah 2011). Fairness mediiert vor allem in frühen Stadien des Prozesses den Effekt von Arbeitsplatzunsicherheit auf affektives Commitment gegenüber der Veränderung (Schumacher et al. 2016). Andererseits kann ein Gefühl von Hilflosigkeit und Mangel an Kontrolle bei der Einwirkung auf Veränderungsprozesse zu emotionalem Rückzug und Symptomen von Depression führen (vgl. Eriksson 2004). In Anbetracht dieser Ergebnisse möchten wir uns in diesem Artikel primär auf prozedurale Gerechtigkeit fokussieren.

In der Literatur wurden verschiedene Regeln beschrieben, die prozedurale Gerechtigkeit determinieren (Leventhal 1980). Wichtig ist das Gefühl von Kontrolle und Beteiligtsein an Prozessen, welches durch Voice (dt. Stimme) erreicht werden kann (Thibaut und Walker 1975). Voice beinhaltet das Einbringen eigener Ideen, Standpunkte oder Vorschläge. Als eine Möglichkeit, eigene Argumente und Sichtweisen zu äußern, können Voice-Interventionen zu verschiedenen positiven Outcomes führen. Im Kontext von organisationalem Wandel zeigten Coch und French (1948), dass Mitarbeiter, die in die Gestaltung von Veränderungsmaßnahmen miteinbezogen wurden, seltener kündigten, bessere Arbeitsleistung zeigten und sich gegenüber Vorgesetzten positiver verhielten, als Mitarbeiter, denen die Veränderung als bereits entschieden kommuniziert wurde. Spätere Studien zeigten, dass die Wahrnehmung prozeduraler Gerechtigkeit in starkem Zusammenhang mit Annäherungsmotivation steht (Murphy und Tyler 2008), und dass das Commitment von Mitarbeitern in Veränderungsprozessen von prozeduraler Gerechtigkeit beeinflusst wird (Lee et al. 2017).

In diesem Artikel möchten wir eingehender untersuchen, welche psychologischen Mechanismen prozedurale Gerechtigkeit auslöst. Im Besonderen möchten wir testen, ob prozedurale Gerechtigkeit dazu führt, dass die mit organisationalem Wandel verbundene Hemmung reduziert und positive Annäherungsmotivation verstärkt werden kann.

\section{Studie 1 - BIS/BAS-Aktivierung und Prozedurale Gerechtigkeit in organisationalem Wandel}

Die Anliegen von Studie 1 sind also folgende: zum einen möchten wir klären, ob organisationaler Wandel wie eine psychologische Bedrohung wirken kann, indem wir BISAktivierung im Rahmen einer Veränderungsmaßnahme erheben. Wir untersuchen, ob diese BIS-Aktivierung von der wahrgenommenen Diskrepanz zwischen Bedürfnissen/ Erwartungen und der Situation vorhergesagt wird. In einem weiteren Schritt wollen wir untersuchen, inwieweit eine Intervention zur Erhöhung prozeduraler Gerechtigkeit BIS-Aktivierung reduzieren und BAS-Aktivierung erhöhen kann. 


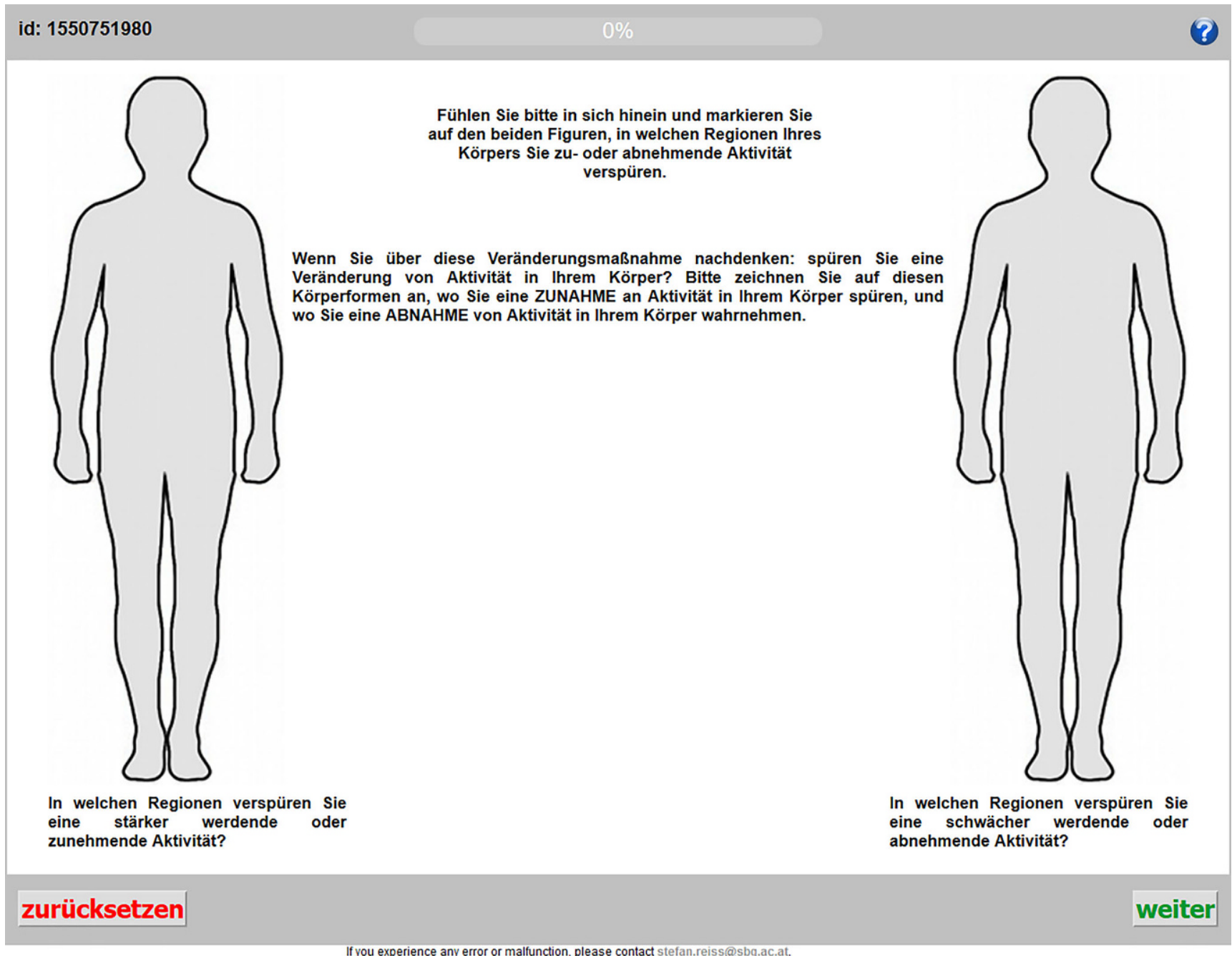

Abb. 2 Darstellung der Körper-Heatmaps in Studie 1

In emotional intensiven Situationen oder bei Bedrohung, wie wir sie im Rahmen organisationalen Wandels vermuten, laufen viele motivational-affektive Prozesse implizit ab, sind also dem Selbstbericht nur schwer zugänglich. Vergangene Forschung zu psychologischer Bedrohung hat gezeigt, dass die Messung von BIS- und BAS-Aktivierung nur eingeschränkt mit expliziten Maßen möglich ist, da selbstberichteter Affekt in Bedrohungssituationen unterdrückt werden kann und daher dem Bewusstsein oft nicht zur Gänze zugängig ist (C. Harmon-Jones et al. 2016). In Studie 1 versuchten wir, diese Probleme auf zwei Weisen zu umgehen: (1) zum einen wurden die Affektmaße retrospektiv erhoben statt in der Situation selbst (vgl. Agroskin et al. 2016; Echebarria-Echabe 2013). Als BIS-Affekt wurde ängstliche Gehemmtheit gemessen, als BAS-Affekt wurde sowohl hoch-aktivierter Affekt (Entschlossenheit) als auch niedrigaktivierter Affekt (Entspannung) gemessen. (2) Zur Validierung des Selbstberichts verwendeten wir eine weitere Methode zur Erfassung von BIS-mediiertem Arousal: so- matotopische Aktivierungskarten (Bodily Sensation Maps; Nummenmaa et al. 2014). Diese „Heatmaps“ körperlicher Aktivierung wurden erfolgreich eingesetzt, um affektive Zustände auf Silhouetten eines menschlichen Körpers zu projizieren. In Studie 1 untersuchten wir, inwiefern wir körperliche Zustände beim Gedanken an die Veränderungsmaßnahmen abbilden können und ob diese mit retroaktiv selbstberichtetem BIS-Affekt zusammenhängen.

\subsection{Methode und Vorgehen}

\subsubsection{Stichprobe}

Für diese Studie wurden $N=76$ berufstätige Versuchspersonen (36 weiblich; 1 fehlende Angabe) aus verschiedenen Unternehmen im Alter von 24 bis 61 Jahren $(M=43,15$, $S D=8,58)$ für eine Onlineumfrage rekrutiert. Im Schnitt waren die VersuchsteilnehmerInnen seit 19,97 Jahren be- 
rufstätig $(S D=9,71)$ und seit 9,31 Jahren $(S D=7,93)$ in ihrem aktuellen Unternehmen angestellt.

Die Versuchspersonen füllten die Studie auf der Onlineplattform Unipark.de aus. Einige Teilnehmer füllten die Umfrage auf mobilen Geräten wie Mobiltelefonen oder Tablets aus, auf welchen das Ausfüllen der Heatmaps meist nur eingeschränkt funktioniert. Um ausreichende Datenqualität zu gewährleisten, wurden deshalb nur Personen in die Analyse aufgenommen, die die Studie an einem Desktopoder Laptop-Computer ausgefüllt hatten.

\subsubsection{Materialien}

Veränderungsprozess Zuerst wurden Versuchspersonen gebeten, an eine Veränderung an ihrem Arbeitsplatz zu denken, die in Kürze ansteht oder aktuell abläuft. Sie sollten sich in die Situation hineinversetzen und über die Details dieser Situation nachdenken. Anschließend wurden sie gebeten, den Veränderungsprozess so genau und detailliert wie möglich zu beschreiben, und mögliche damit verbundene Vor- und Nachteile aufzulisten. Eine explorative Frage erfasste das Stadium der Veränderung, in dem sich die TeilnehmerInnen befanden (siehe Appendix A). Als Manipulationscheck wurden die TeilnehmerInnen gebeten, auf drei 6-stufigen Items anzugeben, wie leicht es ihnen gefallen ist, an eine Veränderungssituation zu denken, diese zu beschreiben, und sich in die Situation hineinzuversetzen $(1=$, ,sehr schwer" $-6=$,sehr leicht“; $M=4,29$; Cronbach's $\alpha=0,86$ ).

Diskrepanzen in der Veränderung Als nächstes wurden die ProbandInnen mittels 6-stufiger Likert-Items befragt, inwiefern das Verhalten der Verantwortlichen in diesem Veränderungsprozess Diskrepanzen darstellte (adaptiert nach Prentice et al. 2019). Die abgefragten Diskrepanzen konnten entweder zwischen eigenen Erwartungen und der Situation entstehen (z.B. „das Verhalten der Verantwortlichen der Veränderung hat mich überrascht"; 5 Items; $M=3,43$; $\alpha=0,76$ ), oder zwischen eigenen Bedürfnissen und der Situation (z.B. ,die Ungerechtigkeit der Veränderungsmaßnahme hat mich verletzt“, 5 Items; $M=4,00 ; \alpha=0,58)^{1}$.

Heatmaps prä-Intervention ProbandInnen bekamen zwei leere Silhouetten von menschlichen Körpern vorgelegt. Sie wurden gebeten, mit der Computermaus auf diesen Silhouetten einzuzeichnen, wo in ihrem eigenen Körper sie eine Zunahme bzw. Abnahme an körperlicher Aktivierung spürten, während sie über die Veränderungssituation nachdachten (siehe Abb. 2).

\footnotetext{
${ }^{1}$ Die Löschung des Items mit der schwächsten Item-Total Korrelation $(r=0,20)$ hätte das Cronbach's Alpha auf $\alpha=0,59$ erhöht, weshalb wir die Skala so beließen wie sie präsentiert wurde.
}

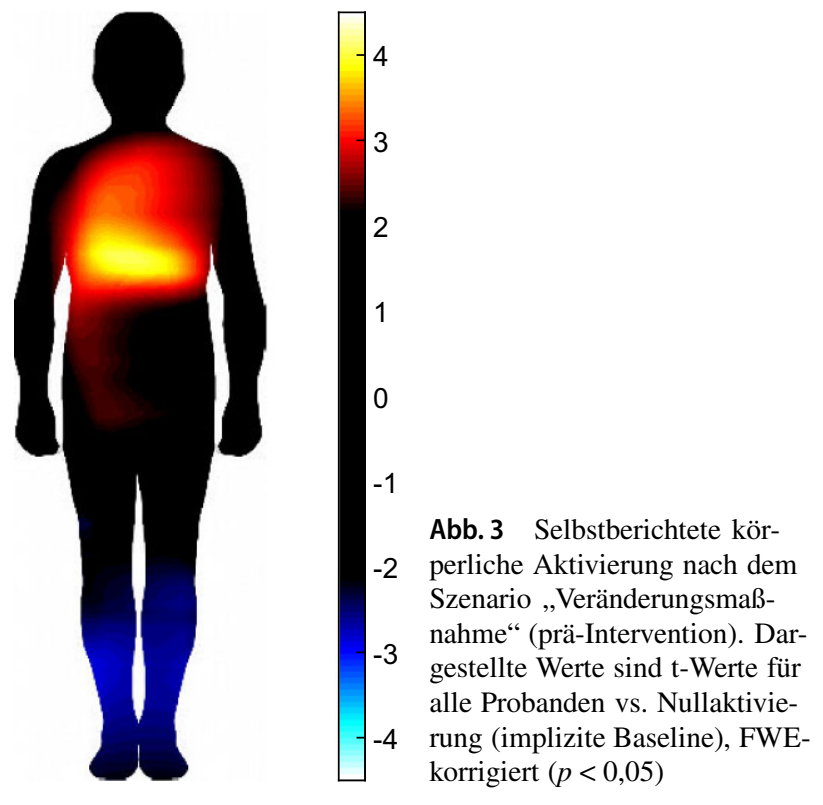

BIS/BAS-Affekt prä-Intervention Mittels 13 Items wurden die Affekt-Facetten BIS, BAS-hohe Aktivierung und BASniedrige Aktivierung erhoben (adaptiert nach Agroskin et al. 2016 und Greenaway et al. 2015). Die Items waren 6 -stufig skaliert $(1=$,überhaupt nicht" $-6=$,sehr"). BISAffekt erfasste ängstliche Gehemmtheit bestand aus fünf Items (z.B. gehemmt, ängstlich, nervös; $\alpha=0,88$ ); BAShohe Aktivierung umfasste fünf Items (z.B. kraftvoll, entschlossen; $\alpha=0,89$ ); BAS-niedrige Aktivierung umfasste drei Items und diente zur Messung eines entspannten, zielkonfliktfreien Zustands (entspannt, ruhig, friedlich; $\alpha=0,73$ ).

Voice/No-Voice Manipulation Zur experimentellen Manipulation von Voice wurde ein eigens kreiertes Szenario verwendet. Personen in der Voice-Bedingung wurden instruiert, sich vorzustellen, dass sie mit einem externen Prozessbegleiter ein individuelles Gespräch führen könnten, um ihre individuellen Bedürfnisse im Veränderungsprozess äußern zu können (Formulierung in Appendix A). In der No-Voice-Bedingung sollten sich die Probanden vorstellen, sie in der Kaffeeküche mit KollegInnen über die Veränderungsmaßnahme auszutauschen. In beiden Bedingungen sollten sie kurz aufschreiben, was sie zur Sprache bringen wollten.

Heatmaps und BIS-BAS-Affekt post-Intervention AnschlieBend füllten die TeilnehmerInnen erneut die Körper-Aktivierungssilhouetten aus und berichteten BIS- $(\alpha=0,88)$, BAS-hoch- $(\alpha=0,92)$ und BAS-niedrig Affekt $(\alpha=0,84)$ wie oben beschrieben. 


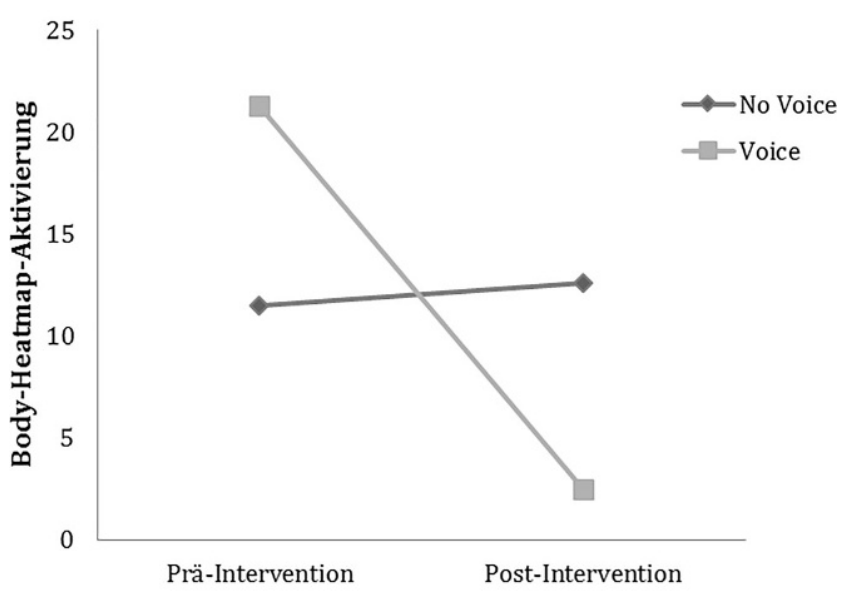

Abb. 4 Index selbstberichteter körperlicher Aktivierung als Funktion von Zeitpunkt (prä- vs. post Intervention) und Versuchsbedingung (Voice vs. No Voice)

Vertrauen Abschließend erhoben wir das Vertrauen der TeilnehmerInnen in die Verantwortlichen der Veränderung als exploratives $\mathrm{Ma}^{2}$. Der Fragebogen umfasste 16 Items auf einer 5-stufigen Likert-Skala ( $1=$,trifft überhaupt nicht zu“ $-5=$,trifft total zu“; $\alpha=0,93$ ). Die Items waren z.B. „Die für die Veränderung Verantwortlichen sind sehr qualifiziert“ oder „Die [...] Verantwortlichen sind um mein Wohl sehr besorgt". Zum Ende der Untersuchung wurden Probanden nach einigen demographischen Daten gefragt.

\subsubsection{Datenaufbereitung}

Körperliche Aktivierung (Heatmaps) Die Körper-Heatmaps wurden mittels Matlab ausgewertet. Die Werte für die Abnahme von Aktivierung wurden pixelweise von den Werten der Zunahme-Heatmap abgezogen. Markierungen auBerhalb der Körper wurden durch eine Maske entfernt. Anschließend wurden die Aktivierungen gegen 0 getestet; zur Kontrolle gegen falsch-positive Aktivierungen wurde auf die Heatmaps eine false-discovery-rate-Korrektur (FDR) mit $\alpha=0,05$ angewandt. Zur Vereinfachung der Datenanalyse wurden die Aktivierungswerte in fünf festgelegten Regionen analysiert: Kopf, Hals, Brust, Bauch und Beine. Neben den einzelnen Regionen untersuchten wir auch ein globales $\mathrm{Ma} \beta$ von körperlicher Aktivierung, in dem wir die Werte der Regionen zu einem Kennwert zusammenfassten. Da die Aktivierung in den Beinen negativ mit den anderen vier Regionen korrelierte, wurde diese Region negativ kodiert in den Kennwert eingerechnet.

\footnotetext{
${ }^{2}$ Wir erhoben außerdem einen für diesen Artikel nicht relevanten Frageboben zur Unterstützung der Maßnahme. Aufgrund eines technischen Problems bei der Administration des Fragebogens umfasst die Stichprobe dieser Skala $n=26$. Da die Stichprobe für zuverlässige Schlussfolgerungen nicht ausreichend groß ist, wird auf die Ergebnisse nicht weiter eingegangen.
}

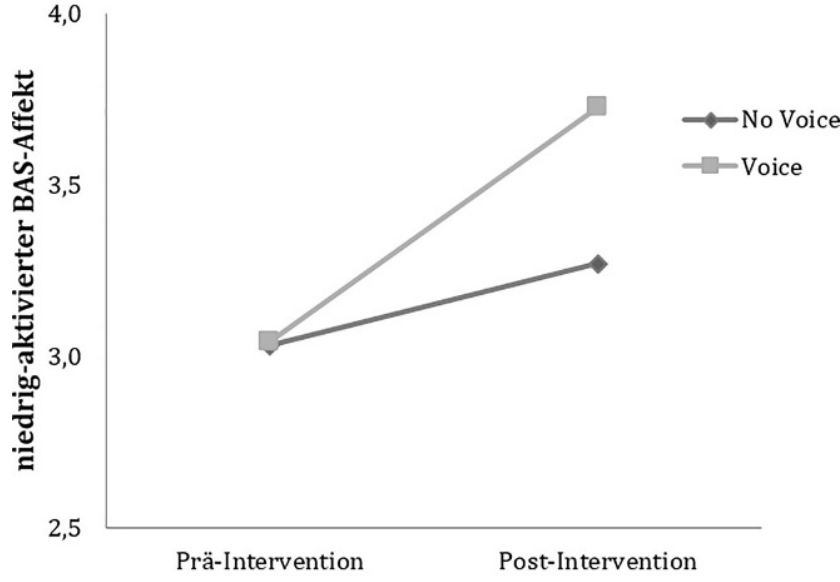

Abb. 5 Niedrig-aktivierter (entspannter) BAS-Affekt als Funktion von Zeitpunkt (prä- vs. post-Intervention) und Versuchsbedingung (Voice vs. No Voice)

\subsection{Ergebnisse}

Heatmaps Zunächst wollten wir untersuchen, ob die Auseinandersetzung mit einer Veränderungsmaßnahme zu einem Anstieg in BIS führt, und ob dies mit den drei AffektFacetten BIS (Ängstlichkeit), BAS-niedrig (Entspannung) und BAS-hoch (Entschlossenheit) korreliert. In der Tat reagierten Probanden auf die Veränderungsmaßnahme mit einer signifikanten Zunahme von Aktivität im Brustbereich, und einer Abnahme von Aktivierung in den Beinen (FWEkorrigierte pixelweise Aktivierung; siehe Abb. 3).

Da die Heatmap-Aktivierung nicht normalverteilt war, werden im folgenden Spearman-Rho-Korrelationskoeffizienten $(\rho)$ berichtet. Das Ausmaß körperlicher Aktivierung korrelierte signifikant negativ mit niedrig aktiviertem BASAffekt $\rho(64)=-0,30, p=0,015$. BIS-Affekt korrelierte marginal mit der Heatmap-Aktivierung, $\rho(64)=0,23, p=0,066$; hochaktivierter BAS-Affekt war nicht mit Heatmap-Aktivierung korreliert, $\rho(64)=-0,14, p=0,27$. Die HeatmapAktivierung zeigte sich also als Indikator für Zunahme von Ängstlichkeit und Abnahme von Entspannung, aber nicht für Entschlossenheit.

Diskrepanz-Erleben und Affekt Im folgenden Schritt untersuchten wir, welchen Einfluss das Erleben von Diskrepanzen auf die Heatmap-Aktivierung sowie BIS- und BAS-Affekt hatten. Erwartungsdiskrepanz und Bedürfnisdiskrepanz korrelierten nicht signifikant miteinander, $r=0,12, p=0,31$. Ein lineares Regressionsmodell, in dem die beiden Skalen Erwartungsdiskrepanz und Bedürfnisdiskrepanz als Prädiktoren für die Heatmap-Aktivierungen eingegeben wurden, erwies sich als signifikant $\left(R^{2}=0,09\right.$; $F(2,63)=3,18, p=0,048)$. Allerdings verfehlten Erwartungsdiskrepanz $(\beta=0,22, t=1,79, p=0,078)$ und Bedürfnisdiskrepanz $(\beta=0,18, t=1,50, p=0,138)$ als einzelne 
Prädiktoren für die Heatmap-Aktivierung statistische Signifikanz. Beide Diskrepanzfacetten ergänzten sich also und wirkten gemeinsam als Prädiktor für körperliches Arousal.

Wir untersuchten außerdem den prädiktiven Effekt der Diskrepanzskalen auf die Affektskalen. BIS-Affekt wurde signifikant von Bedürfnisdiskrepanz vorhergesagt $(\beta=0,36$, $t=3,28, \quad p=0,002)$, aber nicht von Erwartungsdiskrepanz $(\beta=0,08, t=0,70, p=0,49)$, Gesamtmodell $R^{2}=0,14$, $F(2,73)=5,98, p=0,004$. BAS-hochaktivierter Affekt wurde nicht von Diskrepanzen vorhergesagt (beide $t \mathrm{~s}<1,33$, $p>0,18$, Modell $\left.R^{2}=0,05, F(2,73)=1,77, p=0,18\right)$. BASniedrigaktivierter Affekt wurde wiederum durch Bedürfnisdiskrepanz $(\beta=-0,38, t=-3,50, p=0,001)$, aber nicht durch Erwartungsdiskrepanz $(\beta=-0,01, t=-0,05, p=0,96)$ vorhergesagt, Modell $R^{2}=0,15, F(2,73)=6,22, p=0,003$. Somit diente Bedürfnisdiskrepanz, aber nicht Erwartungsdiskrepanz, in der Veränderungssituation als Prädiktor für höhere Ängstlichkeit im Sinne von BIS-Aktivierung und reduziertem entspannten BAS-Affekt.

Voice-Intervention Zur Untersuchung des Einflusses der Voice-Intervention auf Affekt und körperliche Aktivierung wurden die Aktivierungsdaten aller fünf Regionen (Beine rekodiert) in eine ANOVA mit Messwiederholung eingegeben, mit 2 Wiederholungsfaktoren (Heatmap-Region: 5-stufig, Kopf, Hals, Brust, Bauch, Beine; Zeitpunkt: 2-stufig, Prä- und Post-Intervention) und einem Gruppenfaktor (2-stufig, Voice vs. No-Voice). Die Analyse ergab keinen Haupteffekt für Region, $F(2,28,142,1)=1,30$, $p=0,27, \eta_{\mathrm{p}}{ }^{2}=0,022$; einen marginalen Haupteffekt für Zeitpunkt, $F(1,58)=3,29, p=0,075, \eta_{\mathrm{p}}{ }^{2}=0,054$, und keinen signifikanten Effekt von Voice, $F(1,58)=0,00, p=0,98$, $\eta_{\mathrm{p}}{ }^{2}=0,000$. Die für die Fragestellung relevante Interaktion Zeitpunkt $\times$ Voice zeigte jedoch einen signifikanten Effekt, $F(1,58)=4,17, p=0,046, \eta_{\mathrm{p}}{ }^{2}=0,067$; siehe Abb. 4. Während Probanden in der No-Voice-Bedingung keinen Unterschied in der Aktivierung zwischen den beiden Messzeitpunkten zeigten $\left(F(1,58)=0,22, p=0,88, \eta_{\mathrm{p}}{ }^{2}<0,001\right)$, nahm die berichtete Aktivierung in der Voice-Bedingung signifikant ab $\left(F(1,58)=9,28, p=0,003, \eta_{\mathrm{p}}{ }^{2}=0,138\right)$.

BIS-Affekt war nach der Intervention signifikant niedriger als zuvor, $F(1,74)=6,57, p=0,012, \eta_{\mathrm{p}}{ }^{2}=0,082$, jedoch unterschied sich die Veränderung im BIS-Affekt nicht signifikant zwischen der Voice- und der No-Voice-Bedigung (Interaktion Zeitpunkt $*$ Voice $F(1,74)=0,04, p=0,844$, $\left.\eta_{\mathrm{p}}{ }^{2}=0,001\right)$.

Für hochaktivierten BAS-Affekt fand sich ebenfalls ein signifikanter Haupteffekt von Zeitpunkt, da die Werte in beiden Bedingungen anstiegen $(F(1,74)=8,34, p=0,005$, $\left.\eta_{\mathrm{p}}{ }^{2}=0,101\right)$. Das Ausmaß des Anstiegs in BAS-hoch Affekt wurde allerdings nicht signifikant durch die Intervention beeinflusst, $F(1,74)=1,66, p=0,202, \eta_{\mathrm{p}}{ }^{2}=0,022$, auch wenn der Anstieg in der Voice-Bedingung etwas stärker war als in der No-Voice-Bedingung.

Für niedrig-aktivierten BAS-Affekt fand sich ein signifikanter Haupteffekt von Zeit, $\mathrm{F}(1,74)=20,52, p<0,001$, $\eta_{\mathrm{p}}{ }^{2}=0,217$, aber kein Haupteffekt von Voice, $\mathrm{F}(1,74)=1,14$, $p=0,290, \eta_{\mathrm{p}}{ }^{2}=0,015$. Die signifikante Interaktion von Zeitpunkt und Voice auf niedrig-aktivierten BAS-Affekt $\left(F(1,74)=4,73, p=0,033, \eta_{\mathrm{p}}{ }^{2}=0,060\right)$ zeigt an, dass dieser Effekt in der Voice-Bedingung stärker ist als in der NoVoice-Bedingung; siehe Abb. 5. Personen, die prozedurale Gerechtigkeit in der Form von Voice erfahren hatten, zeigten also mehr entspannten BAS-Affekt als Personen, die diese Möglichkeit nicht bekamen.

MitarbeiterInnen, die die Möglichkeit zu Voice hatten, berichteten also nach der Intervention deutlich weniger körperliche Erregung in den Heatmaps und waren entspannter als diejenigen MitarbeiterInnen, die keine Voice-Möglichkeit hatten. Entschlossenheit und Ängstlichkeit entwickelten sich im Vergleich zum Zeitpunkt prä-Intervention (direkt nach der Bedrohung) ebenfalls zum Besseren, wurden allerdings nicht signifikant durch Voice beeinflusst.

Vertrauen Die Analyse des Einflusses der Voice-Intervention auf in die für die Veränderung Verantwortlichen zeigte keinen Effekt. Die Voice-Intervention veränderte also das Vertrauen nicht bedeutsam $(F(1,64)=1,21, p=0,275$, $\left.\eta_{\mathrm{p}}^{2}=0,019\right)$.

\subsection{Diskussion}

In Studie 1 sollte untersucht werden, ob organisationaler Wandel wie eine Bedrohung wirken kann, und ob dieser Bedrohungseffekt durch Diskrepanzerleben erklärt werden kann. In der Tat zeigte sich, dass der Gedanke an organisationalen Wandel zu selbstberichteter körperlicher Aktivierung in Form der Körper-Heatmaps führte. Somit scheinen die Heatmaps ein guter Indikator für ängstliche Gehemmtheit im Sinne von BIS-Aktivierung zu sein. Diese Annahme wird unterstrichen durch die Korrelation der Aktivierungsmappen mit erhöhtem BIS-Affekt (ängstlicher Gehemmtheit) sowie mit der Abnahme von entspanntem BAS-Affekt. Das Erleben von Bedürfnis- und Erwartungsdiskrepanzen sagte die Heatmap-Aktivierung vorher: je stärker die empfundenen Diskrepanzen, desto stärker also die körperlich-affektive Reaktion auf die Veränderungsmaßnahme. Unsere Annahme, dass Diskrepanzerleben im Rahmen von Veränderungsprozessen zu einem Bedrohungszustand führt, konnte also bestätigt werden.

Wir wollten außerdem untersuchen, ob wir durch das Ermöglichen von Voice diesen BIS-Zustand reduzieren und BAS-Aktivierung erhöhen können. Die Intervention zur Erhöhung prozeduraler Gerechtigkeit (Voice) führte zu sig- 
nifikanter Abnahme von körperlicher Aktivierung und zu stärkerer Aktivierung von entspanntem BAS-Affekt.

Diese Ergebnisse illustrieren, dass mit einer vergleichsweise simplen Methode wie der Möglichkeit, individuelle Bedürfnisse zu äußern, negative Auswirkungen von als Bedrohung wahrgenommenen Veränderungsmaßnahmen reduziert werden. Auf unser Fallbeispiel angewandt, könnte man Frau As Angstreaktionen auf die Veränderungsmaßnahme reduzieren, indem man ihr und ihren KollegInnen eine Möglichkeit bietet, eigene Bedürfnisse und Gedanken zu äußern. Durch die Reduktion von körperlichem Arousal und negativem motivationalem Affekt kann sie einfacher aus dem anfänglichen Zustand von Ängstlichkeit und Hemmung in die Handlungsfähigkeit (BAS-Aktivierung) zurückgelangen. Doch lassen sich diese Erkenntnisse noch verfeinern? Reagiert Frau A besonders positiv darauf, wenn ihr die Veränderung als Wachstums-Chance kommuniziert wird? Oder bevorzugt sie Sicherheit und Risikovermeidung?

\section{Studie 2 - Prozedurale Gerechtigkeit und Framing der Veränderungsmaßnahme}

In Studie 2 wollten wir uns eingehender mit dem Einfluss prozeduraler Gerechtigkeit beschäftigen, und welchen Einfluss sie auf Affekt und Motivation hat. Außerdem wollten wir eine weitere Methode untersuchen, um Mitarbeiter in einen Annäherungsmodus zu bekommen: das Framing der Maßnahme in einem Annäherungskontext. Wird eine Maßnahme im Annäherungskontext kommuniziert, stehen Wachstumsziele, potenzielle Gewinne und Chancen im Fokus, was der verhaltensaktivierenden Funktion des BAS entspricht. Im Umkehrschluss liegt das Augenmerk einer im Vermeidungskontext formulierten Veränderungsmaßnahme auf Aspekten wie Arbeitsplatzsicherheit, der Vermeidung von Risiken und Verlusten (vgl. Böhm und Jonas 2016). Diese Formulierung zielt auf das Vermeiden von bedrohlichen Ergebnissen und spricht so die Vermeidungskomponente des BIS an (Jonas et al. 2014). In Studie 2 sollen deshalb Vermeidungs- und Annäherungsframing einer organisationalen Veränderung hinsichtlich ihrer Wirkung auf Verhaltenshemmung (BIS) und Verhaltensaktivierung (BAS) verglichen werden.

Rufen wir uns noch einmal Frau A in Erinnerung und stellen uns vor, dass die Unternehmensleitung sie über die im Zuge des organisationalen Wandels vorgesehene Umstrukturierung des Bezahlungsmodells im Unternehmen informiert. Uns interessiert nun, ob das Formulieren der Veränderung mit Wachstumszielen (,Leistung lohnt sich wieder" oder ,Förderung einer engagierten [...] Arbeitsweise") zu stärkerer Annäherungsmotivation führt als die Formu- lierung mit Vermeidungszielen (z. B. ,Vermeiden, dass sich Leistung nicht mehr lohnt").

Laut der Theorie des Regulatorischen Fokus (Higgins 1997) können sich Personen hinsichtlich ihrer motivationalen Orientierung unterscheiden, mit der sie Ziele verfolgen. Personen mit Promotion-Fokus streben eher nach Wachstum und sehen Chancen in Annäherungszielen, während Personen mit Prevention-Fokus eher nach Sicherheit streben und Ziele eher vermeidungsorientiert formulieren, zum Beispiel als Vermeiden von Risiken oder potenziellen Verlusten (vgl. Böhm und Jonas 2016).

Wir würden also einen besonders starken Motivationsgewinn durch ein Annäherungsframing der Maßnahme erwarten, wenn Frau A einen stärkeren Promotion- als Prevention-Fokus hat, da hier eine motivationale Passung zwischen regulatorischem Fokus und Framing bestehen würde (sog. regulatorischer Fit; Higgins 2000).

In Studie 2 verwendeten wir die Linien-Bisektionsaufgabe (line bisection task, LBT; Jewell und McCourt 2000) als Indikator für Annäherungs-/Vermeidungsmotivation (d.h. BAS und BIS). LBT ist ein objektives, nonverbales, implizites Maß für asymmetrische präfrontale Aktivität. ProbandInnen versuchen hierbei, horizontale Linien exakt zu halbieren. Halbierungsfehler mit Tendenz nach links wurden als Indikator für eine stärkere asymmetrische Aktivierung im rechten präfrontalen Kortex und somit für stärkere BIS-Aktivierung und Vermeidungsmotivation validiert. Fehlertendenzen nach rechts indizieren hingegen stärkere asymmetrische Aktivierung im linken präfrontalen Kortex und BAS-Aktivierung (Annäherungsmotivation) (Agroskin et al. 2016; Nash et al. 2011). Außerdem erhoben wir die Bereitschaft von ProbandInnen, mit BIS und BAS verbundene Verhaltensweisen zu zeigen.

\subsection{Methode und Vorgehen}

\subsubsection{Stichprobe}

Für Studie 2 wurden $N=127$ berufstätige Versuchspersonen aus verschiedenen Unternehmen über Internetforen und direkte Anfragen an Unternehmen für eine Onlineumfrage rekrutiert. Die meisten Versuchspersonen arbeiteten in der Dienstleistungsbranche $(n=47)$ und in technischen Berufen $(n=29)$. Die Versuchspersonen füllten die Studie auf der Onlineplattform Unipark.de aus. Zwei Versuchspersonen wurden aus der Analyse ausgeschlossen, da sie die Fragen zum Manipulationscheck falsch beantworteten. Die finale Stichprobe bestand aus 125 deutschsprachigen Personen (64 weiblich) im Alter von 19 bis 68 Jahren $(M=39,91$, $S D=12,39)$. Im Schnitt waren die Versuchspersonen seit 11,28 Jahren $(S D=10,29)$ in ihrem aktuellen Unternehmen angestellt. Abhängig von der Bedingung wurden die Ver- 
suchspersonen in vier Gruppen randomisiert geteilt $\left(n_{1}=33\right.$, $n_{2}=30, n_{3}=30, n_{4}=32$ ).

\subsubsection{Materialien}

Anfang der Studie Nach einer kurzen Begrüßung füllten die Versuchspersonen den Händigkeitsfragebogen ${ }^{3}$ (9 Items; $\alpha=0,87$; Oldfield 1971) und die erste Linien-Bisektionsaufgabe (der LBT) aus, die als eine Baseline analysiert wurde $(\alpha=0,69)$. Die Versuchspersonen wurden instruiert, acht abgebildete horizontale Linien möglichst genau in der Mitte zu teilen. Die Versuchspersonen wurden darauf hingewiesen, dabei keine Hilfsmittel zu verwenden und den markierten Mittelpunkt nicht mehr zu ändern. Höhere Werte indizieren einen stärkeren Rechts-Bias und somit Annäherungsmotivation (BAS-Aktivierung), niedrigere Werte deuten auf Vermeidungsmotivation (BIS-Aktivierung) hin.

Als Nächstes bearbeiteten sie eine Bedürfnisskala (Jamieson et al. 2010) und eine Skala zum regulatorischen Fokus (Sassenberg et al. 2012). Die 7-stufigen Items ( $1=$,trifft

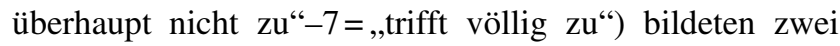
Subskalen: eine zum Annäherungs-Fokus (z. B. „Ich bin risikobereit“; 12 Items; $\alpha=0,87$ ) und eine zum VermeidungsFokus (z.B. „Ich halte mich eigentlich immer an Regeln und Vorschriften“; 8 Items; $\alpha=0,64$ ).

Voice und Framing Anschließend bekamen die Versuchspersonen eines von vier Szenarien zum Lesen (je nach der Gruppe). Die Versuchspersonen sollten sich in die beschriebene Situation so gut wie möglich hineinversetzen. Die Szenarien wurden speziell für diese Studie entwickelt und dienten der Manipulation der untersuchten Interventionen: Gerechtigkeit (Voice vs. No-Voice) und Framing der Veränderungsmaßname (Annäherung vs. Vermeidung). Die Kombination der beiden Manipulationen ergab vier Szenarien (Voice * Annäherung, No-Voice* Annäherung, Voice * Vermeidung und No-Voice* Vermeidung). Die Szenarien bestanden aus einer fiktiven E-Mail von der Unternehmensleitung. In allen vier Szenarien wurden die Versuchspersonen als Angestellte des Unternehmens angesprochen und darauf hingewiesen, dass der Vorstand des Unternehmens plane, ein neues leistungsbasiertes Bezahlungsmodell in der Organisation zu implementieren. Je nach Framing-Bedingung wurde dargestellt, welche Resultate durch das neue Entlohnungsmodell erreicht (Annäherung) oder vermieden (Vermeidung) werden sollten (z.B. „Leistung lohnt sich wieder" erreichen oder „Leistung lohnt sich nicht mehr“

\footnotetext{
${ }^{3}$ Der Händigkeitsfragebogen wurde präsentiert, da mit der Händigkeit assoziierte Unterschiede in der Lateralisierung des Frontallappens einen Einfluss auf die Ergebnisse der Linien-Bisektionsaufgabe haben können. Die Daten von Linkshändern wurden für die Analyse der Linien-Bisektionsaufgabe ausgeschlossen (vgl. Agroskin et al. 2016; McGregor et al. 2013; Nash et al. 2010).
}

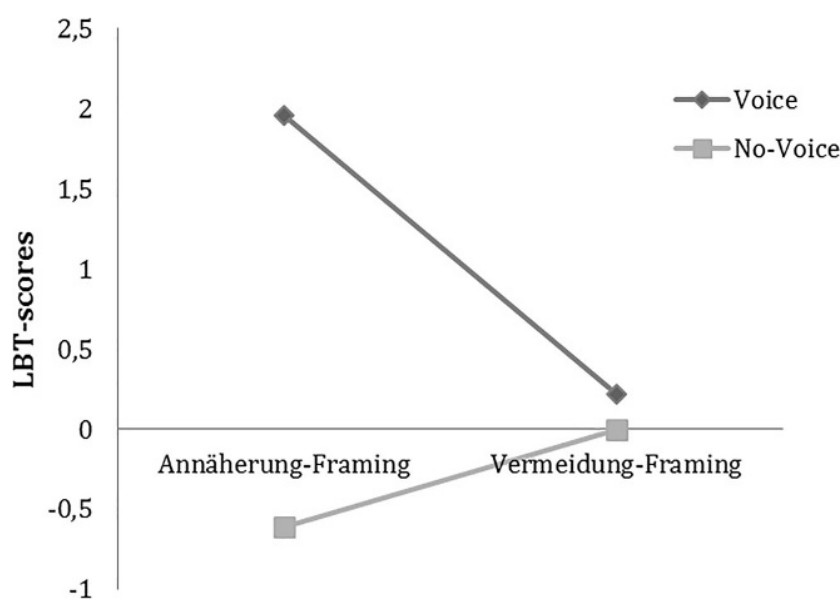

Abb. 6 Line Bisection Task-Scores als Funktion von Gerechtigkeit (Voice vs. No-Voice) und Framing (Annäherung vs. Vermeidung)

vermeiden). Zusätzlich wurden die Kernaussagen mittels entweder grüner Häkchen (Annäherung) oder roter Kreuzchen (Vermeidung) für die Mitarbeiter visualisiert.

In der Voice-Bedingung wurde zusätzlich mitgeteilt, dass die Mitarbeiter nach drei Monaten die Möglichkeit haben würden, Rückmeldung zum eingeführten Bezahlungsmodell zu geben und Argumente zu äußern, die aus ihrer Sicht für oder gegen das Entlohnungsmodell sprächen. In der NoVoice-Bedingung entfiel dieser Hinweis. Die Szenarien sind in Appendix B dargestellt.

Linien-Bisektionsaufgabe (LBT) Sofort nach dem Szenario bearbeiteten die Versuchspersonen zum zweiten Mal den LBT $(\alpha=0,79)$. Die Versuchspersonen bekamen die gleiche Instruktion wie beim ersten LBT.

BIS/BAS-Affekt Wie in Studie 1 wurden die Affekt-Facetten BIS, BAS-hohe Aktivierung und BAS-niedrige Aktivierung erhoben. Die Items waren 5-stufig skaliert $(1=$,überhaupt nicht" $-5=$,sehr"). BIS-Affekt umfasste fünf Items (z.B. ängstlich, nervös, gehemmt; $\alpha=0,93$ ); BAS-hohe Aktivierung umfasste vier Items (z.B. kraftvoll, entschlossen; $\alpha=0,68)$; BAS-niedrige Aktivierung umfasste zwei Items (entspannt, ruhig, friedlich; $r=0,43, p<0,001$ ).

Verhalten Im Folgenden bearbeiteten die Versuchspersonen zwei für diesen Artikel irrelevante Skalen ${ }^{4}$. AnschlieBend beantworteten die Versuchspersonen auf einer 5-stu-

\footnotetext{
${ }^{4}$ Die Versuchspersonen füllten zum zweiten Mal die Bedürfnisskala von Jamieson et al. (2010) aus. Zusätzlich bewerteten die Versuchspersonen die Einführung des neuen Entlohnungsmodells mithilfe von einer Skala, die nach Jonas und Steindl (2015) adaptiert wurde. Obwohl diese Skala $(\alpha=0,68)$ die Annäherungs-/Vermeidungsreaktionen nicht untersucht, wurde sie aus exploratorischen Gründen analysiert. Die Analyse ergab aber keine signifikante Ergebnisse, alle $p s>0,05$. Die analysierte Skala ist im Appendix B dargestellt.
} 
Tab. 1 Regulatorischer Fit als Prädiktor von niedrig-aktiviertem BASAffekt

\begin{tabular}{lllll}
\hline Prädiktor & $b$ & $S E$ & $t$ & $p$ \\
\hline Konstante & 2,96 & 0,09 & 33,34 & $<0,001$ \\
Regulatorischer Fit & 0,03 & 0,17 & 0,19 & 0,851 \\
Framing & $-0,33$ & 0,18 & $-1,88$ & 0,063 \\
Fit * Framing & $-1,04$ & 0,35 & $-2,99$ & 0,003 \\
\hline
\end{tabular}

figen Skala $(1=$, überhaupt nicht“ $-5=$, ,sehr"), wie wahrscheinlich bestimmte Verhaltensweisen in Reaktion auf die Einführung des neuen Bezahlungsmodells für sie wären (adaptiert nach Jonas und Steindl 2015; Traut-Mattausch et al. 2011). Die Verhaltensweisen gliederten sich in positive Annäherung (6 Items; z. B. „Ich werde bald versuchen, meine Arbeitsleistung zu steigern"; $\alpha=0,74$ ), negative Annäherung (6 Items; z. B. „Ich werde den Verantwortlichen mit Protest drohen"; $\alpha=0,89$ ) und Vermeidung (7 Items; z. B. „Ich werde mich im Betrieb einfach weniger engagieren“; $\alpha=0,65)$. Die Skala ist im Appendix B dargestellt.

Am Ende der Studie füllten die Versuchspersonen eine 5-Stufige Skala ( $1=$,überhaupt nicht" $-5=$,sehr"; $\alpha=0,82$ ) zu ihren Gedanken bezüglich der Veränderungsmaßnahme aus (adaptiert nach Jonas und Steindl 2015; Traut-Mattausch et al. 2011). Diese Skala wurde aus exploratorischen Gründen hinzugefügt. ${ }^{5}$ Abschließend gaben die Versuchspersonen einige demographische Daten an und beantworteten drei Fragen zum Manipulationscheck, in dem nach einigen Details des Szenarios gefragt wurde.

\subsubsection{Datenaufbereitung}

LBT Der LBT-Bias wurde berechnet, indem die Anzahl der linken Abweichungen von den rechten Abweichungen subtrahiert wurde (McGregor et al. 2010, Studie 1). Niedrige LBT-Scores indizieren daher einen Anstieg in Vermeidungsmotivation, höhere Scores indizieren einen Anstieg in Annäherungsmotivation.

Regulatorischer Fit Um zu untersuchen, ob motivationale Passung die Effektivität des Annäherungsframings beeinflusste, berechneten wir den regulatorischen Fit als Passung zwischen dem regulatorischen Fokus (Promotion vs. Prevention) als Persönlichkeitsmerkmal und dem Framing der Veränderungsmaßname (Annäherung vs. Vermeidung). Der dominante regulatorische Fokus der Versuchspersonen wurde in Anlehnung an Lockwood et al. (2002, Studie 3) berechnet, indem der Differenzwert aus Annäherungs- und

\footnotetext{
5 Obwohl diese Skala die Annäherungs-/Vermeidungsreaktionen nicht im Fokus hat, wurde sie aus exploratorischen Gründen analysiert. Die Analyse ergab aber keine signifikante Ergebnisse, alle $p s>0,269$. Die analysierte Skala ist im Appendix B dargestellt.
}

Tab. 2 Regulatorischer Fit als Prädiktor von BIS-Affekt

\begin{tabular}{lllll}
\hline Prädiktor & $b$ & $S E$ & $t$ & $p$ \\
\hline Konstante & 2,43 & 0,10 & 24,91 & $<0,001$ \\
Regulatorischer Fit & $-0,09$ & 0,20 & $-0,47$ & 0,637 \\
Framing & 0,38 & 0,20 & 1,95 & 0,053 \\
Fit * Framing & 0,75 & 0,39 & 1,90 & 0,060 \\
\hline
\end{tabular}

Vermeidungsscore gebildet wurde. Versuchspersonen mit positivem Differenzwert wurden dem Promotion-Fokus zugeordnet; Versuchspersonen, deren Differenzwert negativ war, wurden dem Prevention-Fokus zugeordnet. Um die Variable „Regulatorischer Fit“ zu erstellen, wurden der dominante Fokus und das zufällig zugeteilte Framing-Szenario verglichen.

\subsection{Ergebnisse}

Das Ziel von Studie 2 war die Hypothese zu testen, dass Interventionen wie Gerechtigkeit und/oder das Framing der Maßnahme im Annäherungskontext helfen können, Mitarbeiter in einen konstruktiven Annäherungsmodus zu bekommen. Der Annäherungs- vs. Vermeidungszustand wurde mithilfe von LBT, Affektskala und Verhaltensskala gemessen.

LBT Um für mit der Händigkeit assoziierten hemisphärischen Unterschiede zu kontrollieren (z.B. Drake und Myers 2006), analysierten wir nur die Daten von rechtshändigen TeilnehmerInnen $\left(n_{v^{*} \text { an }}=31, n_{\mathrm{nv}^{*} \text { an }}=28, n_{v^{*} \text { ver }}=26\right.$, $n_{\mathrm{nv} * \mathrm{ver}}=31$ ), die durch die Händigkeitsskala identifiziert wurden. Die 2 (Gerechtigkeit: Voice vs. No-Voice) $\times 2$ (Framing: Annäherung vs. Vermeidung) ANCOVA mit dem ersten LBT als Kovariate ergab einen signifikanten Haupteffekt von Voice, $F(1,111)=5,80, p=0,018$,

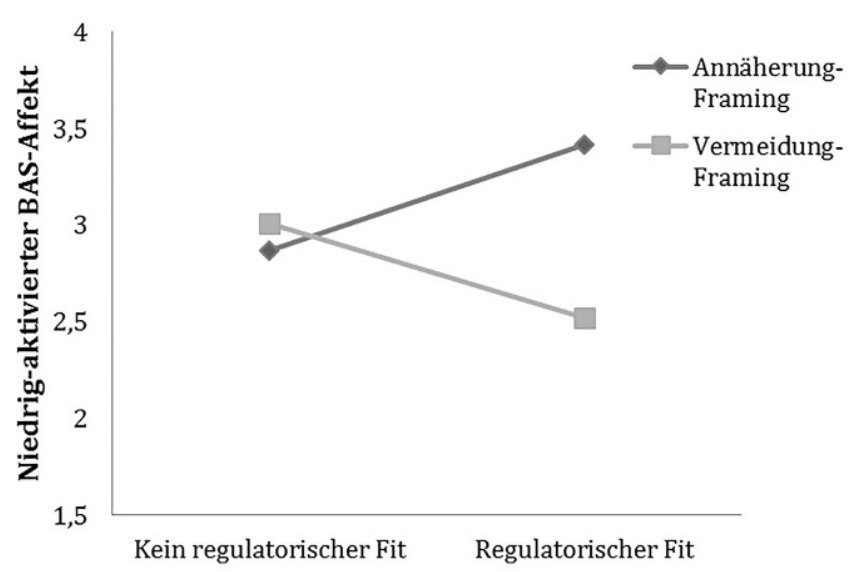

Abb. 7 Niedrig-aktivierter BAS-Affekt als Funktion von Framing (Annäherung vs. Vermeidung) und regulatorischem Fit (Fit vs. Kein Fit) 


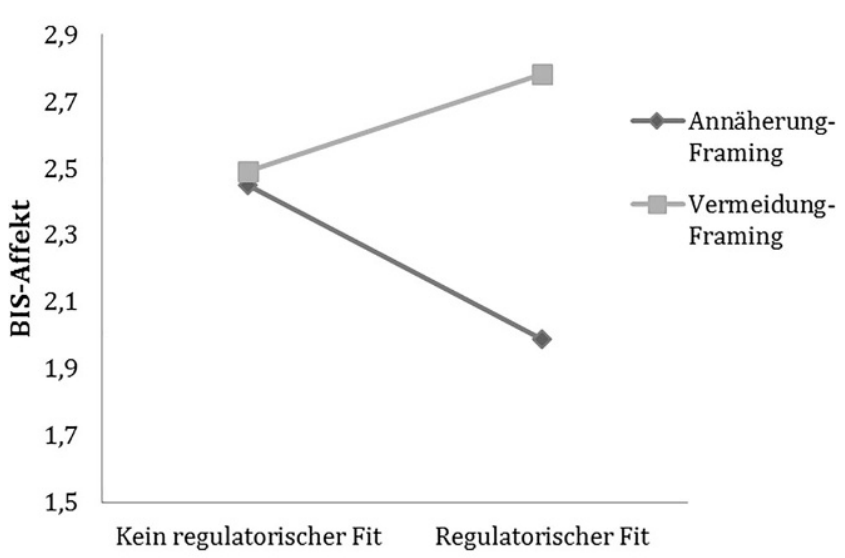

Abb. 8 BIS-Affekt als Funktion von Framing (Annäherung vs. Vermeidung) und regulatorischem Fit (Fit vs. Kein Fit)

$\eta_{\mathrm{p}}{ }^{2}=0,050$. Wenn die Versuchspersonen eine Voice-Möglichkeit hatten, zeigten sie höhere LBT-Scores (stärkere Annäherungsmotivation bzw. niedrigere Vermeidungsmotivation) als wenn sie keine solche Möglichkeit hatten $\left(M_{\mathrm{v}}=1,40, S D_{\mathrm{v}}=3,99, M_{\mathrm{nv}}=-0,53, S D_{\mathrm{nv}}=3,90\right)$. Dieser Effekt wurde durch eine signifikante Gerechtigkeit $\times$ Framing Interaktion ergänzt, $F(1,111)=4,02, p=0,047, \eta_{\mathrm{p}}{ }^{2}=0,035$ (Abb. 6). Der Unterschied zwischen der Voice- und der NoVoice-Bedingung war signifikant bei Annäherungsframing $(p=0,002)$, aber nicht bei Vermeidungsframing $(p=0,795)$. Wenn MitarbeiterInnen die Möglichkeit hatten, Voice zu äußern, zeigten sie erhöhte neurobehaviorale Muster, die mit Annäherungsmotivation verbunden sind; dieses Muster zeigte sich besonders stark in Verbindung mit Annäherungsframing der Veränderungsmaßnahme, nicht aber bei Vermeidungsframing.

BIS/BAS-Affekt Um zu untersuchen, ob die Voice-Intervention und das Annäherungsframing der Veränderungsmaßnahme positiven Einfluss auf Affekt nehmen, wurden die Daten von allen 125 Versuchspersonen analysiert. Bezüglich des BIS-Affekts zeigte die $2 \times 2$ ANOVA einen signifikanten Haupteffekt von Voice, $F(1,121)=3,99$, $p=0,048, \eta_{\mathrm{p}}{ }^{2}=0,032$, und von Framing, $F(1,121)=3,99$, $p=0,048, \eta_{\mathrm{p}}{ }^{2}=0,032$. Versuchspersonen, die eine VoiceMöglichkeit hatten, fühlten weniger BIS-Affekt (Ängstlichkeit), als Versuchspersonen, die keine Möglichkeit für Voice hatten $\left(M_{\mathrm{v}}=2,22, S D_{\mathrm{v}}=1,07, M_{\mathrm{nv}}=2,61, S D_{\mathrm{nv}}=1,06\right)$. Darüber hinaus berichteten die Versuchspersonen, die das annäherungsformulierte Szenario lasen, weniger negativen BIS-Affekt, als die Versuchspersonen, die das vermeidungsformulierte Szenario lasen $\left(M_{\mathrm{an}}=2,22, S D_{\mathrm{an}}=1,07\right.$, $\left.M_{\text {ver }}=2,61, S D_{\text {ver }}=1,06\right)$. Die Voice $\times$ Framing Interaktion war nicht signifikant $(p=0,689)$.

Bezüglich des hoch-aktivierten BAS-Affekts (Entschlossenheit) wurden keine signifikanten Effekte gefunden, alle $p s>0,432$. Bezüglich des niedrig-aktivierten BAS-Affekts (Entspannung) ergab die $2 \times 2$ ANOVA einen marginalen Haupteffekt von Framing, $F(1,121)=3,32, p=0,071$, $\eta_{\mathrm{p}}{ }^{2}=0,027$. Wenn die Veränderungsmaßnahme im Annäherungskontext präsentiert wurde, berichteten die Versuchspersonen tendenziell mehr entspannten BAS-Affekt als bei Vermeidungsframing $\left(M_{\mathrm{an}}=3,15, S D_{\mathrm{an}}=0,91, M_{\mathrm{ver}}=2,81\right.$, $\left.S D_{\text {ver }}=1,08\right)$. Der Effekt von Voice $(p=0,105)$ sowie die Interaktion $(p=0,985)$ waren nicht signifikant.

Insgesamt unterstützen die Ergebnisse unsere Erwartungen: die Formulierung der Veränderung im Annäherungskontext reduzierte Ängstlichkeit und erhöhte entspannten BAS-Affekt und Annäherungsmotivation (LBT), vor allem in Verbindung mit prozeduraler Gerechtigkeit. Allerdings fand sich kein Effekt von Voice oder Framing auf Entschlossenheit (BAS-hochaktiviert).

Verhalten Als Nächstes wurde die Auswirkung der Interventionen auf Annäherungs- und Vermeidungsverhalten analysiert. Hinsichtlich der positiven Annäherung und Vermeidung wurden keine signifikante Unterschiede zwischen den Bedingungen gefunden, alle $p s>0,178$. Die $2 \times 2$ ANOVA ergab aber einen marginalen Haupteffekt von Framing hinsichtlich der negativen Annäherung, $F(1,121)=3,88, p=0,051, \eta_{\mathrm{p}}{ }^{2}=0,031$. Die Versuchspersonen, die ein annäherungs-formuliertes Szenario lasen, tendierten also dazu, weniger Bereitschaft zum aktiven Vorgehen gegen die Veränderungsmaßnahme zu berichten $\left(M_{\mathrm{an}}=1,86, S D_{\mathrm{an}}=0,81, M_{\mathrm{ver}}=2,16, S D_{\mathrm{ver}}=0,90\right)$.

Regulatorischer Fit Abschließend wurde der regulatorische Fit als Moderator analysiert. Wir erwarteten, dass die Effekte von Annäherungs- und Vermeidungsframing stärker wirken, wenn die Formulierung mit dem persönlichen motivationalen Fokus von MitarbeiterInnen übereinstimmt (sog. regulatorischer Fit). Für die Analyse wurde das PROCESSMacro für SPSS (Model 1; Hayes 2017) verwendet. Als abhängige Variablen wurden diejenigen Variablen analysiert, die vom Framing signifikant oder marginal beeinflusst wurden, nämlich BIS-Affekt, entspannter BAS-Affekt und negatives Annäherungsverhalten (aktives Vorgehen gegen die Maßnahme). Die Analyse ergab eine signifikante Framing $\times$ Fit Interaktion bezüglich des entspannten BAS-Affekts, siehe Tab. 1, und eine marginale Interaktion bezüglich des BIS-Affekts, siehe Tab. 2. Versuchspersonen empfanden mehr Entspannung und tendenziell weniger ängstliche Gehemmtheit, wenn sie das annäherungsformulierte Szenario lasen. Dies war aber nur bei Personen mit annäherungsorientiertem regulatorischem Fokus (Promotion) der Fall, also wenn regulatorischer Fit gegeben war (siehe Abb. 7 und 8). Regulatorischer Fit hatte keinen Einfluss auf die Intention, gegen die Veränderungsmaßnahme vorzugehen (negatives Annäherungsverhalten; $p=0,518$ ). 
Unsere zusätzliche Analyse zeigte also, dass die Passung zwischen motivationalem Fokus und Framing der Maßnahme nur bei MitarbeiterInnnen mit Promotion-Fokus zu mehr positiven Ergebnissen führte. MitarbeiterInnen mit Prevention-Fokus blieben hingegen ängstlich gehemmt und zeigten wenig Entspannung, wenn sie mit der Veränderungsmaßnahme konfrontiert wurden. Hier half es auch nicht, wenn die Maßnahme ein Annäherungsframing hatte.

\subsection{Diskussion}

Zusammenfassend ergänzte Studie 2 die erste Studie mit folgenden Erkenntnissen: Die Untersuchung der LinienBisektionsaufgabe (LBT) als neurobehaviorales Maß für die Veränderung von Annäherungs- und Vermeidungsmotivation zeigte, dass die Verbindung von zwei Interventionen besonders effektiv war. Wenn Voice gegeben war und die Veränderungsmaßnahme im Annäherungskontext präsentiert wurde, zeigten VersuchteilnehmerInnen erhöhte LBT-Scores, was einen Anstieg von Annäherungsmotivation beziehungsweise Abhahme von Vermeidungsmotivation indiziert. Mittels der affektiven Maße lässt sich das Ergebnis feiner aufschlüsseln: Prozedurale Gerechtigkeit als Intervention (Voice) führte zu einer Abnahme von ängstlichem BIS-Affekt in Reaktion auf Veränderungsmaßnahmen, besonders in Verbindung mit Annäherungsframing der Maßnahme. Außerdem führte Annäherungsframing tendenziell zu mehr Entspannung (niedrig-aktiviertem BAS-Affekt) und weniger negativem Annäherungsverhalten (Protest gegen die Maßnahme). Somit zeigte sich, dass prozedurale Gerechtigkeit und Annäherungsframing vor allem zu Angstreduktion führten.

Weitere Analysen zeigten auch die Wichtigkeit, regulatorischen Fokus als Persönlichkeitsmerkmal zu berücksichtigen: Annäherungsframing wirkte vor allem bei MitarbeiterInnen mit Promotion-Fokus (Annäherung als dominanter regulatorischer Fokus), aber weniger gut bei MitarbeiterInnen mit Prevention-Fokus.

\section{Generelle Diskussion}

Die vorgestellten Studien untersuchten organisationalen Wandel im Kontext sozialpsychologischer Bedrohungsforschung. Wir konnten Belege für die Annahme finden, dass organisationaler Wandel motivational-affektive Effekte hat, die denen klassischer psychologischer Bedrohung ähnlich sind (Jonas et al. 2014), nämlich körperliches Arousal (Studie 1), erhöhter BIS-Affekt (z.B. Ängstlichkeit; Studie 1 und 2) sowie eine Abnahme von Annäherungsmotivation (Studie 2). Unsere Forschung zeigt aber auch Wege auf, um MitarbeiterInnen aus diesem Bedrohungszustand zu bringen (Studie 1), oder das Ausmaß des Bedrohungszu- stands schon bei der Entstehung zu reduzieren (Studie 2): durch prozedurale Fairness (Voice) konnten wir negativen Affekt, Vermeidungsmotivation und körperliches Arousal bei MitarbeiterInnen senken und Annäherungsmotivation verstärken. Wir fanden außerdem positive Effekte bei annäherungsorientiertem Framing der Maßnahme, vor allem bei MitarbeiterInnen mit Promotion-Fokus. Als sekundäre Erkenntnisse der Studie konnten wir in Studie 1 die KörperHeatmaps und in Studie 2 den LBT als implizite Maße für das Ausmaß eines proximalen Bedrohungszustands validieren, der mit BIS-Affekt und Abnahme von entspanntem BAS-Affekt zusammenhängt.

Die beschriebenen Ergebnisse haben wichtige theoretische Implikationen. Vorige Studien haben festgestellt, dass organisationaler Wandel für Mitarbeiter ein Stressor sein kann, der negative Emotionen hervorruft (Eriksson 2004). Unsere Studien bestätigten diese Annahmen und erklären die psychologischen Mechanismen hinter diesen Prozessen. Organisationaler Wandel kann für MitarbeiterInnen eine stress-induzierende Bedrohung darstellen, indem er ein Gefühl von Diskrepanz und Ängstlichkeit auslöst, welches negative Auswirkung auf Motivation hat. Wenn MitarbeiterInnen sich bedroht fühlen, erleben sie Vermeidungsmotivation. Dies bedeutet im Kontext von organisationalem Wandel, dass sie bei der Konfrontation mit einem Veränderungsprozess Angst und Unsicherheit empfinden, sowie alarmiert und gehemmt sind. Diese Hemmung erschwert es ihnen, sich an neue Prozesse anzupassen, gewohnte Verhaltensweisen zu ändern und die Veränderungsmaßnahme zu unterstützen.

Unsere Studien demonstrierten jedoch auch, dass psychologische Interventionen wie das Schaffen prozeduraler Gerechtigkeit negative motivational-affektive Auswirkungen von Veränderungsprozessen abschwächen können. Wenn MitarbeiterInnen gerecht behandelt werden und das Gefühl haben, dass ihre Bedürfnisse berücksichtigt wurden, erleben sie weniger Diskrepanzen zwischen eigenen Bedürfnissen und Realität. Sie nehmen Veränderungsprozesse als weniger bedrohlich wahr, empfinden weniger Angst und sind eher bereit, diese Prozesse zu unterstützen. Prozedurale Gerechtigkeit hat positive Auswirkungen, sowohl auf affektiver als auch auf motivationaler Ebene. In einer Metaanalyse von 2013 betrachteten Colquitt et al. (2013) den Zusammenhang zwischen Gerechtigkeit und Emotionen und merkten an, dass Affekt lange Zeit keine zentrale Rolle in der Entwicklung der Theorie zu Gerechtigkeit gespielt hatte. Außerdem wurde meist keine Unterscheidung zwischen annäherungs- und vermeidungsbezogenem Affekt getätigt. Unsere Studie berücksichtigte diese Kritikpunkte und zeigte, dass prozedurale Gerechtigkeit positive Reaktionen fördert, indem sie ängstliche Gehemmtheit (BIS-Aktivierung) reduziert und motivationale Annäherung (BASAktivierung) erhöht. Diese Ergebnisse erweitern unser Ver- 
ständnis bezüglich der positiven Auswirkungen prozeduraler Gerechtigkeit.

Unsere Ergebnisse haben mehrere Implikationen in der Arbeitswelt: zum einen müssen sich Entscheidungsträger bewusst sein, dass Veränderungsmaßnahmen, so sinnvoll sie aus ökonomischer Sicht sein mögen, bei MitarbeiterInnen im Unternehmen zu einem negativen motivationalaffektiven Zustand führen können. Der Bedrohungszustand entsteht aus wahrgenommenen Diskrepanzen zwischen der Situation und persönlichen Bedürfnissen sowie Erwartungen. Je stärker diese Diskrepanzen, desto intensiver die Bedrohung und die motivational-affektive Reaktion darauf.

Um diese Wirkung zu reduzieren, können verschiedene Maßnahmen ergriffen werden. Zum einen haben wir gezeigt, wie wichtig prozedurale Gerechtigkeit für MitarbeiterInnen ist. Deshalb ist es von fundamentaler Bedeutung, Möglichkeiten zu eröffnen, an einem Veränderungsprozess mitzuwirken - oder zumindest eigene Meinungen beitragen zu können, die auch angehört werden. Um im Rahmen einer Umstrukturierung negative motivationale Konsequenzen bei Frau A zu reduzieren, könnten bereits in frühen Stadien der Veränderung Maßnahmen ergriffen werden: Das Einrichten von Foren, in denen Meinungen und Vorschläge eingebracht werden können, schafft Voice und erhöht bei Frau A das Gefühl, aktiv am Prozess mitwirken und mitbestimmen zu können. Auch können externe Prozessbegleiter an der Veränderung teilnehmen, die während des gesamten Prozesses Feedback aufnehmen und vermitteln.

Eine weitere Methode, die wir in diesem Manuskript skizzierten, ist das Framing der Veränderungsmaßnahme in einen Annäherungskontext. Auf Affektebene zeigten sich folgende Ergebnisse: Durch die Einbettung der Maßnahme in den zielorientierten Rahmen kann BIS-aktivierte ängstliche Gehemmtheit reduziert und BAS-Affekt erhöht werden. Dies trifft vor allem auf MitarbeiterInnen mit PromotionFokus zu, wenn regulatorischer Fit vorhanden ist. Im Umkehrschluss suggerieren unsere Daten jedoch auch, dass ein regulatorischer Fit zwischen Prevention-Fokus und Vermeidungsframing der Maßnahme keinen Anstieg von BAS-Affekt bewirkt. Ein Fokus auf das Vermeiden von negativen Konsequenzen von Stagnation scheint also hinderlich für erfolgreiche Veränderung zu sein.

Auf Ebene der Verhaltensintentionen zeigte sich, dass alle ProbandInnen, auch diejenigen mit Prevention-Fokus, besser auf annäherungsorientiertes Framing reagierten. Auch für die Linien-Bisektionsaufgabe zeigten sich positive Effekte von Annäherungsframing. Interessanterweise zeigten Personen mit Prevention-Fokus niedrigere Vermeidungsmotivation und weniger negative Verhaltensintentionen nach der annäherungsorientierten Formulierung der Maßnahme. Annäherungsframing wirkte für vermeidungsorientierte MitarbeiterInnen also vor allem auf verhaltensbezogene Maße, aber nicht auf affektive Zustände.
Eine einfache Lösung, um vermeidungsorientierte MitarbeiterInnen direkt in einen positiven affektiven Zustand zu bringen, ergibt sich aus unserer Studie folglich nicht. Um Ängstlichkeit bei prevention-orientierten MitarbeiterInnen zu reduzieren, ist es ratsam, Handlungsschritte konkret zu definieren und Wenn-Dann-Pläne zu formulieren. So könnten sicherheitsorientierte MitarbeiterInnen in ihrem Zustand von Arousal und Verhaltenshemmung in einen Handlungszustand zu kommen (Böhm 2018). Sobald diese motivationale Reorientierung stattgefunden hat, sind MitarbeiterInnen eher in der Lage, sich auf aktives Verhalten einzulassen. Dieser Vorschlag (vgl. Böhm und Jonas 2016; Böhm et al. 2017) sollte in zukünftiger Forschung noch ausführlicher untersucht werden.

\section{Limitationen und zukünftige Forschung}

Neben den spannenden Erkenntnissen unserer Forschung gibt es Limitationen, auf die wir eingehen wollen: Die Generalisierbarkeit unserer Erkenntnisse unterliegt - wie viele Fragebogenstudien - einigen Einschränkungen. Studie 1 untersuchte echte Veränderungsprozesse bei berufstätigen Personen verschiedener Unternehmen und in unterschiedlichen Hierarchie-Ebenen. Insofern waren die Veränderungsmaßnahmen durchaus heterogen. In Studie 2 untersuchten wir berufstätige Personen und präsentierten ihnen das gleiche Veränderungsszenario, um die experimentelle Kontrolle zu erhöhen. Die kann allerdings den Nachteil mit sich bringen, dass die Szenarien nicht realistisch genug sind, um tiefgehende emotionale und motivationale Konflikte auszulösen. Trotz dieser Limitationen lieferten die beiden Studien interessante und informative Ergebnisse zu unserer Fragestellung. Zukünftige Forschung könnte in einer größer angelegten Studie in einem echten Unternehmen Maße von Motivation, Affekt, und körperlichem Stress über den gesamten Verlauf einer Veränderungsmaßnahme beobachten, und welche Rolle prozedurale Gerechtigkeit dort spielt.

Ein weiterer Kritikpunkt für Studie 1 steht in Verbindung mit der Heterogenität der Veränderungsmaßnahmen: zu verschiedenen Zeitpunkten einer Maßnahme kann prozedurale Gerechtigkeit unterschiedlich stark Einfluss auf Motivation und Affekt von MitarbeiterInnen haben (Schumacher et al. 2016). Da unsere Stichprobe nicht ausreichend groß für zuverlässige Aussagen über einen solchen Effekt war (und nicht zentral für unsere Fragestellung), sollte sich zukünftige Forschung auch auf verschiedene Stadien von Veränderung konzentrieren.

Zukünftige Forschung sollte sich auch der anderen Facetten organisationaler Gerechtigkeit annehmen: In den hier berichteten Studien untersuchten wir den Einfluss prozeduraler Gerechtigkeit auf Indikatoren bedrohungsrelevanter Variablen. Unsere Studien können keine Aussage über den 
Einfluss prozeduraler Gerechtigkeit im Vergleich mit informationaler, distributiver oder interpersonaler Gerechtigkeit treffen, oder darüber, welche der Facetten am wichtigsten ist. Die Effekte zeigen aber, dass prozedurale Gerechtigkeit ein wichtiger Faktor im Veränderungsprozess sein kann. Weitere Forschung sollte sich dem Zusammenspiel der verschiedenen Facetten von Gerechtigkeit widmen und weitere Interventionen zu den jeweiligen Gerechtigkeitsdimensionen untersuchen.

\section{Fazit}

Die durchgeführten Studien zeigen, dass schon das Hineinversetzen in Situationen, in denen MitarbeiterInnen prozedurale Gerechtigkeit erfahren, Vermeidung und negativer Affekt reduzieren kann. Dies unterstreicht das Potenzial prozeduraler Gerechtigkeit als aktivierender und motivierender Faktor. Im Gegensatz zu erlebter Ungerechtigkeit, die zur Ängstlichkeit und Vermeidung führt (Prentice et al. 2019), fördert prozedurale Gerechtigkeit ein positives und konstruktives Verhalten - eine wichtige Basis für erfolgreichen organisationalen Wandel.

Danksagung Wir danken Natalie Schreitter, MSc., für ihren wertvollen Beitrag zur Konzeptualisierung und Durchführung von Studie 1.

Funding Open access funding provided by Austrian Science Fund (FWF).

Open Access Dieser Artikel wird unter der Creative Commons Namensnennung 4.0 International Lizenz (http://creativecommons.org/ licenses/by/4.0/deed.de) veröffentlicht, welche die Nutzung, Vervielfältigung, Bearbeitung, Verbreitung und Wiedergabe in jeglichem Medium und Format erlaubt, sofern Sie den/die ursprünglichen Autor(en) und die Quelle ordnungsgemäß nennen, einen Link zur Creative Commons Lizenz beifügen und angeben, ob Änderungen vorgenommen wurden.

\section{Literatur}

Agroskin, D., Jonas, E., Klackl, J., \& Prentice, M. (2016). Inhibition underlies the effect of high need for closure on cultural closedmindedness under mortality salience. Frontiers in Psychology, 7, 1583. https://doi.org/10.3389/fpsyg.2016.01583.

Al-Haddad, S., \& Kotnour, T. (2015). Integrating the organizational change literature: a model for successful change. Journal of Organizational Change Management, 28, 234-262. https://doi.org/ 10.1108/JOCM-11-2013-0215.

Amodio, D. M., Master, S. L., Yee, C. M., \& Taylor, S.E. (2008). Neurocognitive components of the behavioral inhibition and activation systems: implications for theories of self-regulation. Psychophysiology, 45, 11-19. https://doi.org/10.1111/j.1469-8986.2007. 00609.x.

Angus, D. J., \& Harmon-Jones, E. (2016). On the neuroscience of approach and withdrawal motivation, with a focus on the role of asymmetrical frontal cortical activity. Psychophysiology, 19, 37-63. https://doi.org/10.1108/S0749-742320160000019003.
Balogun, J., \& Hailey, V.H. (2008). Exploring strategic change. London: Pearson Education. https://doi.org/10.1007/s13398-0140173-7.2.

Blascovich, J. (2008). Challenge and threat appraisal. Handbook of Approach and Avoidance Motivation, 432-444. https://doi.org/10. 4324/9780203888148.ch25.

Böhm, A.M. (2018). Zwischen Sicherheit und Wachstum: Ansätze zur Überbrückung und Integration motivationaler Grundorientierungen in Führung, Coaching und Beratung. Doctoral Dissertation. Salzburg: University of Salzburg.

Böhm, A. M., \& Jonas, E. (2016). Der regulatorische Fokus als Führungsinstrument für eine Bedürfnisorientierte und effektive Mitarbeiterkommunikation. Gruppe. Interaktion. Organisation. Zeitschrift Für Angewandte Organisationspsychologie (GIO), 47, 171-179. https://doi.org/10.1007/s11612-016-0316-8.

Böhm, A.M., Mühlberger, C., \& Jonas, E. (2017). Wachstums- und sicherheitsorientierung im coaching. In S. Greif, H. Möller \& W. Scholl (Hrsg.), Handbuch Schlüsselkonzepte im Coaching (S. 1-9). Berlin, Heidelberg: Springer. https://doi.org/10.1007/ 978-3-662-45119-9_98-1.

Burnes, B. (2009). Management change: a strategic approach to organisational dynamics. London: Pearson Education.

Coch, L., \& French Jr, J. R. P. (1948). Overcoming resistance to change. Human Relations, 1, 512-532.

Cohen-Charash, Y., \& Spector, P.E. (2001). The role of justice in organizations: a meta-analysis. Organizational Behavior and Human Decision Processes, 86, 278-321. https://doi.org/10.1006/obhd. 2001.2958.

Colquitt, J.A. (2001). On the dimensionality of organizational justice: a construct validation of a measure. Journal of Applied Psychology, 86(3), 386-400. https://doi.org/10.1037/0021-9010.86.3.386.

Colquitt, J. A., Conlon, D.E., Wesson, M. J., Porter, C. O. L.H., \& Ng, Y.K. (2001). Justice at the millenium: a meta-analytic review of 25 years of organizational justice research. Journal of Applied Psychology, 86, 425-445. https://doi.org/10.1037//0021-9010.86. 3.425 .

Colquitt, J. A., Scott, B. A., Rodell, J.B., Long, D. M., Zapata, C.P., Conlon, D.E., \& Wesson, M. J. (2013). Justice at the millennium, a decade later: a meta-analytic test of social exchange and affectbased perspectives. Journal of Applied Psychology, 98, 199-236. https://doi.org/10.1037/a0031757.

Corr, P. J. (2004). Reinforcement sensitivity theory and personality. Neuroscience and Biobehavioral Reviews, 28, 317-332. https:// doi.org/10.1016/j.neubiorev.2004.01.005.

Drake, R., \& Myers, L. (2006) Visual attention, emotion, and action tendency: Feeling active or passive. Cognition \& Emotion, 20, 608-622.

Echebarria-Echabe, A. (2013). Mortality salience and uncertainty: similar effects but different processes? European Journal of Social Psychology, 43, 185-191. https://doi.org/10.1002/ejsp.1938.

Eriksson, C. B. (2004). The effects of change programs on employees' emotions. Personnel Review, 33, 110-126. https://doi.org/10. 1108/00483480410510642.

Folger, R., Skarlicki, D. P., \& Change, O. (1999). Unfairness and resistance to change: hardship as mistreatment-proquest psychology journals-proquest. Journal of Organizational Change Management, $12,35-50$.

Fuchs, S. (2011). The impact of manager and top management identification on the relationship between perceived organizational justice and change-oriented behavior. Leadership and Organization Development Journal, 32, 555-583. https://doi.org/10.1108/ 01437731111161067.

Fugate, M., Prussia, G. E., \& Kinicki, A. J. (2012). Managing employee withdrawal during organizational change: the role of threat appraisal. Journal of Management, 38, 890-914. https://doi.org/10. $1177 / 0149206309352881$.

Gray, J. A. (1981). A critique of eysenck's theory of personality. In H. J. Eysenck (Hrsg.), A model for personality (S. 246-276). Berlin, 
Heidelberg: Springer. https://doi.org/10.1007/978-3-642-67783$0 \_8$.

Gray, J. A., \& McNaughton, N. (2000). The neuropsychology of anxiety: an enquiry into the functions of the septo-hippocampal system (2. Aufl.). Oxford: Oxford University Press. https://doi.org/ 10.1017/S0140525X00013066.

Greenaway, K.H., Storrs, K.R., Philipp, M.C., Louis, W.R., Hornsey, M. J., \& Vohs, K.D. (2015). Loss of control stimulates approach motivation. Journal of Experimental Social Psychology, 56, 235-241. https://doi.org/10.1016/j.jesp.2014.10.009.

Harmon-Jones, E., \& Allen, J. J. B. (1998). Anger and frontal brain activity: eeg asymmetry consistent with approach motivation despite negative affective valence. Journal of Personality and Social Psychology, 74, 1310-1316. https://doi.org/10.1037/0022-3514.74.5. 1310.

Harmon-Jones, C., Bastian, B., \& Harmon-Jones, E. (2016). Detecting transient emotional responses with improved self-report measures and instructions. Emotion, 16, 1086-1096. https://doi.org/10. 1037/emo0000216.

Harmon-Jones, E., Gable, P.A., \& Peterson, C.K. (2010). The role of asymmetric frontal cortical activity in emotion-related phenomena: a review and update. Biological Psychology, 84, 451-462. https://doi.org/10.1016/j.biopsycho.2009.08.010.

Hayes, A. F. (2017). Introduction to mediation, moderation, and conditional process analysis: A regression-based approach. New York, NY: Guilford Press.

Helpap, S., \& Bekmeier-Feuerhahn, S. (2016). Employees' emotions in organizational change: a new angle on the sensemaking approach. Academy of Management Proceedings, 2016, 16730. https://doi.org/10.5465/ambpp.2016.16730abstract.

van den Heuvel, M., Demerouti, E., \& Bakker, A. B. (2014). How psychological resources facilitate adaptation to organizational change. European Journal of Work and Organizational Psychology, 23, 847-858. https://doi.org/10.1080/1359432X.2013. 817057.

Higgins, E. T. (1997). Beyond pleasure and pain. American Psychologist, 52, 1280-1300. https://doi.org/10.1037/0003-066X.52.12. 1280.

Higgins, E. T. (2000). Making a good decision: value from fit. American Psychologist, 55, 1217-1230. https://doi.org/10.1037/0003066X.55.11.1217.

Jacobs, G., Van Witteloostuijn, A., \& Christe-Zeyse, J. (2013). A theoretical framework of organizational change. Journal of Organizational Change Management, 26, 772-792. https://doi.org/10. 1108/JOCM-09-2012-0137.

Jamieson, J.P., Harkins, S. G., \& Williams, K. D. (2010). Need threat can motivate performance after ostracism. Personality and Social Psychology Bulletin, 36, 690-702. https://doi.org/10.1177/ 0146167209358882.

Jewell, G., \& McCourt, M.E. (2000). Pseudoneglect: a review and meta-analysis of performance factors in line bisection tasks. Neuropsychologia, 38, 93-110.

Jonas, E., \& Steindl, C. (2015). Annäherung oder vermeidung? eine motivationale sicht auf reformprozesse. Personal Quarterly, 15, $10-16$.

Jonas, E., McGregor, I., Klackl, J., Agroskin, D., Fritsche, I., Holbrook, C., Quirin, M., et al. (2014). Threat and defense: from anxiety to approach. Advances in Experimental Social Psychology, 49, 219-286. https://doi.org/10.1016/B978-0-12-800052-6.00004-4.

Kiefer, T. (2002). Understanding the emotional experience of organizational change: evidence from a merger. Advances in Developing Human Resources, 4, 39-61. https://doi.org/10.1177/ 1523422302004001004

Klonek, F.E., Güntner, A. V., Lehmann-Willenbrock, N., \& Kauffeld, S. (2015). Using motivational interviewing to reduce threats in conversations about environmental behavior. Frontiers in Psychology, 6, 1-16. https://doi.org/10.3389/fpsyg.2015.01015.
Leana, C. R., \& Barry, B. (2000). Stability and change as simultaneous experiences in organizational life. The Academy of Management Review, 25, 753-759.

Lee, K., Sharif, M., Scandura, T., Kim, J., Lee, K., \& Sharif, M. (2017). Procedural justice as a moderator of the relationship between organizational change intensity and commitment to organizational change. Journal of Organizational Change Management, 30, 501-524. https://doi.org/10.1108/JOCM-08-2015-0139.

Leventhal, G. S. (1980). What should be done with equity theory? new approaches to the study of fairness in social relationships. In K. Gergen, M. Greenberg \& R. Willis (Hrsg.), Social exchange: advances in theory and research (S. 27-55). New York: Plenum Press. https://doi.org/10.1007/978-1-4613-3087-5.

Lewis, L. (2019). Organizational change: creating change through strategic communication. Hoboken, NJ: Wiley-Blackwell.

Lockwood, P., Jordan, C. H., \& Kunda, Z. (2002). Motivation by positive or negative role models: regulatory focus determines who will best inspire us. Journal of Personality and Social Psychology, 83, 854. https://doi.org/10.1037/0022-3514.83.4.854.

McGregor, I., Nash, K., Mann, N., \& Phills, C.E. (2010). Anxious uncertainty and reactive approach motivation (ram). Journal of Personality and Social Psychology, 99, 133.

McGregor, I., Prentice, M., \& Nash, K. (2013). Anxious uncertainty and reactive approach motivation (RAM) for religious, idealistic, and lifestyle extremes. Journal of Social Issues, 69, 537-563. https://doi.org/10.1111/josi.12028.

Murphy, K., \& Tyler, T. (2008). Procedural justice and compliance behaviour: the mediating role of emotions. European Journal of Social Psychology, 38, 652-668. https://doi.org/10.1002/ejsp.502.

Nash, K., McGregor, I., \& Inzlicht, M. (2010). Line bisection as a neural marker of approach motivation. Psychophysiology, 47, 979-983. https://doi.org/10.1111/j.1469-8986.2010.00999.x.

Nash, K., McGregor, I., \& Prentice, M. (2011). Threat and defense as goal regulation: from implicit goal conflict to anxious uncertainty, reactive approach motivation, and ideological extremism. Journal of Personality and Social Psychology, 101, 1291-1301. https:// doi.org/10.1037/a0025944.

Nummenmaa, L., Glerean, E., Hari, R., \& Hietanen, J. K. (2014). Bodily maps of emotions. Proceedings of the National Academy of Sciences, 111, 646-651. https://doi.org/10.1073/pnas. 1321664111.

Oldfield, R.C. (1971). The assessment and analysis of handedness: the Edinburgh inventory. Neuropsychologia, 9, 97-113.

Palmer, I., Dunford, R., Buchanan, D. A., \& Jabri, M. (2017). Managing organizational change (3. Aufl.). New York, NY: McGrawHill.

Prentice, L., Jonas, E., \& Prentice, M. (2019). Injustice as a threat: injustice arouses motivational discrepancies that drive passive avoidance and anxiety. Manuscript in Preparation.

Proost, K., Verboon, P., \& Van Ruysseveldt, J. (2015). Organizational justice as buffer against stressful job demands. Journal of Managerial Psychology, 30, 487-499. https://doi.org/10.1108/JMP-022013-0040.

Sassenberg, K., Ellemers, N., \& Scheepers, D. (2012). The attraction of social power: the influence of construing power as opportunity versus responsibility. Journal of Experimental Social Psychology, 48, 550-555. https://doi.org/10.1016/j.jesp.2011.11.008.

Scholz, T.M. (2015). The human role within organizational change: a complex system perspective. In Change management and the human factor (S. 19-31). Berlin Heidelberg: Springer.

Schumacher, D., Schreurs, B., Van Emmerik, H., \& De Witte, H. (2016). Explaining the relation between job insecurity and employee outcomes during organizational change: a multiple group comparison. Human Resource Management, 55, 809-827. https:// doi.org/10.1002/hrm.21687.

Shah, N. (2011). A study of the relationship between organisational justice and employee readiness for change. Journal of Enterprise 
Information Management, 24, 224-236. https://doi.org/10.1108/ 17410391111122835.

Sittenthaler, S., Steindl, C., \& Jonas, E. (2015). Legitimate vs. illegitimate restrictions - a motivational and physiological approach investigating reactance processes. Frontiers in Psychology. https:// doi.org/10.3389/fpsyg.2015.00632.

Steindl, C., \& Jonas, E. (2015). The dynamic reactance interaction-how vested interests affect people's experience, behavior, and cognition in social interactions. Frontiers in Psychology, 6, 1-16. https://doi.org/10.3389/fpsyg.2015.01752.

Stouten, J., Rousseau, D. M., \& De Cremer, D. (2018). Successful organizational change: integrating the management practice and scholarly literatures. Academy of Management Annals, 12, 752-788. https://doi.org/10.5465/annals.2016.0095.

Thibaut, J.W., \& Walker, L. (1975). Procedural justice: a psychological analysis. Hillsdale, NJ: Lawrence Erlbaum.

Traut-Mattausch, E., Jonas, E., Schwennen, C., \& Peus, C. (2011). Things will get better: führt der optimismus in den erfolg von steuerreformen zu weniger widerstand, mehr vertrauen und einer positiveren einstellung? Wirtschaftspsychologie, 13, 43-52.

Viswesvaran, C., \& Ones, D.S. (2002). Examining the construct of organizational justice: a meta-analytic evaluation of relations with work attitudes and behaviors. Journal of Business Ethics, 38, 193-203. https://doi.org/10.1023/A:1015820708345.

Vuori, T., \& Virtaharju, J. (2012). On the role of emotional arousal in sensegiving. Journal of Organizational Change Management, 25, $48-66$.

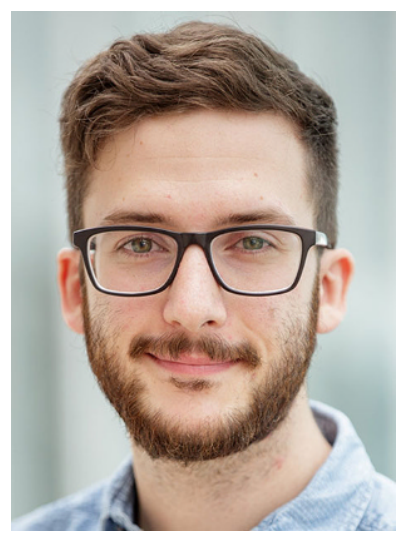

Stefan Reiss ist Doktorand in der Abteilung Sozialpsychologie an der Universität Salzburg. In seiner Dissertation befasst er sich mit den unmittelbaren Auswirkungen psychologischer Bedrohung auf Emotion und Motivation. Er erforscht außerdem physiologische Prozesse im zentralen und peripheren Nervensystem, die diese Effekte begleiten.

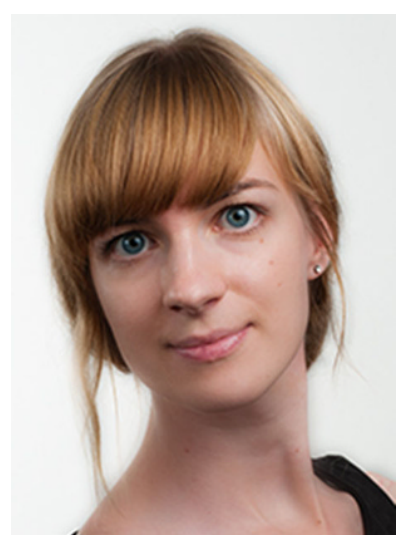

Dr. Liza Prentice hat in der Abteilung Sozialpsychologie an der Universität Salzburg promoviert. In ihrer Dissertation befasste sie sich mit der Frage, wie sich Menschen aus verschiedenen Kulturen in ihren Reaktionen auf erlebte Ungerechtigkeit unterscheiden. Ihr aktueller Interessenschwerpunkt liegt auf der Erforschung interkultureller Kompetenzen und effektiver interkultureller Kommunikation.

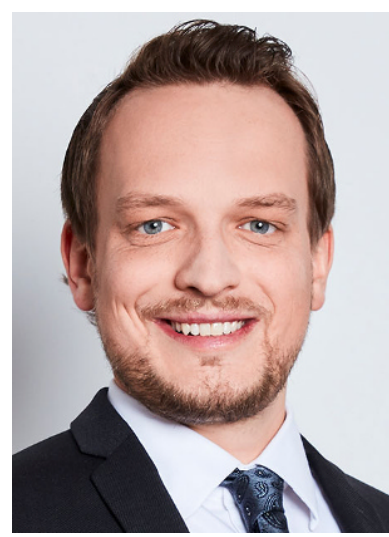

Christoph Schulte-Cloos absolvierte sein Masterstudium der Psychologie mit Schwerpunkt auf Sozial- und Organisationspsychologie an der Universität Salzburg. Aktuell ist er im privatwirtschaftlichen Umfeld im Bereich Personal- und Organisationsentwicklung tätig und beschäftigt sich insbesondere mit der gezielten Förderung und Entwicklung von zukunftsrelevanten Kompetenzen bei MitarbeiterInnen und Führungskräften.

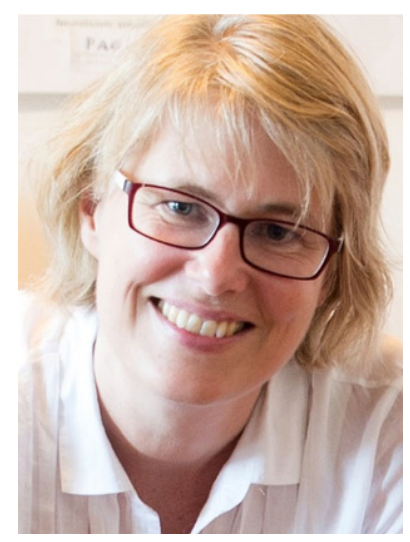

Prof. Dr. Eva Jonas ist Professorin und Leiterin der Abteilung Sozialpsychologie und des Fachbereichs Psychologie an der Universität Salzburg. In ihrer Forschung beschäftigt sie sich mit dem Umgang mit Bedrohungen und sozialen Interaktionsprozessen (z. B. Berater-Klienten Interaktionen, Gerechtigkeit). Außerdem ist sie Leiterin des Universitätslehrganges „Supervision, Mediation, Coaching" der Universität Salzburg und des ,Masters in Training and Development" an der Salzburg Management Business School. 